\title{
Mono-specific algal diets shape microbial networking in the gut of the sea urchin Tripneustes gratilla elatensis
}

\author{
Matan Masasa ${ }^{1,2}$, Ariel Kushmaro ${ }^{3}$, Esti Kramarsky-Winter ${ }^{3}$, Muki Shpigel ${ }^{4}$, Roy Barkan ${ }^{1,2}$, Alex Golberg ${ }^{5}$, \\ Abraham Kribus ${ }^{6}$, Nadav Shashar ${ }^{1}$ and Lior Guttman ${ }^{2^{*}}$ (D)
}

\begin{abstract}
Background: Algivorous sea urchins can obtain energy from a diet of a single algal species, which may result in consequent changes in their gut microbe assemblies and association networks.

Methods: To ascertain whether such changes are led by specific microbes or limited to a specific region in the gut, we compared the microbial assembly in the three major gut regions of the sea urchin Tripneustes gratilla elatensis when fed a mono-specific algal diet of either Ulva fasciata or Gracilaria conferta, or an algal-free diet. DNA extracts from 5 to 7 individuals from each diet treatment were used for Illumina MiSeq based 16S rRNA gene sequencing (V3-V4 region). Niche breadth of each microbe in the assembly was calculated for identification of core, generalist, specialist, or unique microbes. Network analyzers were used to measure the connectivity of the entire assembly and of each of the microbes within it and whether it altered with a given diet or gut region. Lastly, the predicted metabolic functions of key microbes in the gut were analyzed to evaluate their potential contribution to decomposition of dietary algal polysaccharides.
\end{abstract}

Results: Sea urchins fed with U. fasciata grew faster and their gut microbiome network was rich in bacterial associations (edges) and networking clusters. Bacteroidetes was the keystone microbe phylum in the gut, with core, generalist, and specialist representatives. A few microbes of this phylum were central hub nodes that maintained community connectivity, while others were driver microbes that led the rewiring of the assembly network based on diet type through changes in their associations and centrality. Niche breadth agreed with microbes' richness in genes for carbohydrate active enzymes and correlated Bacteroidetes specialists to decomposition of specific polysaccharides in the algal diets.

Conclusions: The dense and well-connected microbial network in the gut of Ulva-fed sea urchins, together with animal's rapid growth, may suggest that this alga was most nutritious among the experimental diets. Our findings expand the knowledge on the gut microbial assembly in T. gratilla elatensis and strengthen the correlation between microbes' generalism or specialism in terms of occurrence in different niches and their metabolic arsenal which may aid host nutrition.

*Correspondence: lior.guttman@mail.huji.ac.il

${ }^{2}$ Israel Oceanographic and Limnological Research, The National Center for Mariculture, P.O. Box 1212,8811201 Eilat, Israel

Full list of author information is available at the end of the article

\section{Background}

The ability of microbes to colonize different niches, i.e., their niche breadth, determines the composition and therefore networking of the microbial assembly when challenged by various environmental forces [1]. Generalist microbes are capable of occupying various niches, original author(s) and the source, provide a link to the Creative Commons licence, and indicate if changes were made. The images or other third party material in this article are included in the article's Creative Commons licence, unless indicated otherwise in a credit line to the material. If material is not included in the article's Creative Commons licence and your intended use is not permitted by statutory regulation or exceeds the permitted use, you will need to obtain permission directly from the copyright holder. To view a copy of this licence, visit http://creativecommons.org/licenses/by/4.0/. 
while specialists are those with a narrower habitat, i.e., found in fewer niches. Specialization can also be determined as unique if the microbe appears consistently only in a particular niche, condition or occasion [2, 3]. With the constant development of new tools for analyzing microbial communities, specific platforms for network analyses have also emerged. These tools allow the discrimination of association networks following their topologies as to density, clustering rate, diameter, length of shortest path, and other indices [4]. Accordingly, the organizational level of the community can be highly random, small-world, or scale-free $[4,5]$. In random networks, the connectivity of microbe nodes is not easily disrupted since many microbial nodes can be reached by any other node through a short number of steps, and the associating nodes are more likely to be neighbors of each other [6]. A closer investigation into the different nodes in an assembly enables us to reveal the key hub microbes with the highest centrality and connectivity, as demonstrated by a fair number of edges (associations) and their proximity to other microbe nodes [7]. Likewise, driver microbes with the greatest contribution to the rewiring of a network under a certain weight (i.e., niche) can be identified by the changes in their associating members, type of association, and centrality [8].

Among numerous environmental forces, diet is considered to influence the composition of the gut-microbe assembly (GMA) of various aquatic invertebrates [9]. A particularly strong effect may be attained if animals are restricted to only one particular nutrient source. A prime example is sea urchins that can gain their energy from a single, usually preferred, algal species $[10,11]$. In many cases such preference has been credited with positive impacts on performance, e.g., growth, gonad color and somatic index $[12,13]$.

Evidence that sea urchins that can be cultured when fed different macroalgae, each as a sole energy source, are favorable for study of the responses of GMA and microbial networking to changes in diet. Toward this goal we selected the sea urchin Tripneustes gratilla elatensis as a model algivorous species to examine whether and how diet restriction to a single species of alga, either Ulva fasciata (Chlorophyta) or Gracilaria conferta (Rhodophyta), will affect the gut microbial assembly compared to a plant-rich but algal-depleted pelleted diet. Not only changes in the composition and networking that could occur at the level of the entire assembly were considered, but also specific changes in the associations and centrality in the network of specific microbes in the assembly. It was further hypothesized that such changes may be more prominent in one of the three major regions in the gut (esophagus, stomach, or intestine) rather than over the entire organ. Hence, the experiment aimed at identification of the key microbes or microbe taxa in the gut of T. gratilla elatensis through their generalism or specialism in the different niches examined (diet types and gut regions) as per their occurrence in broader or narrower niches and also their content of genes for carbohydrates decomposition as potential contribution in feed metabolism.

\section{Methods}

\section{Sea urchin cultivation with experimental diets}

Fifty-four 6-month-old individuals of Tripneustes gratilla elatensis from a single hatch at the National Center for Mariculture (NCM, Eilat, Israel), were assigned for the feeding trial in an in-house experimental system which allows control of environmental conditions including the recommended ambient light for studies of this species [14]. This system comprises nine 90L rectangular tanks and allows maintenance of water quality, temperature, and oxygen saturation via a rapid flow of fresh filtered sea water, as well as continuous aeration of the tanks. Prior to restricting the diet to a given experimental feed treatment, all sea urchins were fed the algal diet of Ulva fasciata together with Gracilaria conferta recommended at the local integrated multi-trophic aquaculture system (IMTA). After two weeks of acclimation, homogeny in the physical parameters of body ('test') diameter $(85.2 \pm 2 \mathrm{~mm})$ and weight $(272.4 \pm 17 \mathrm{~g}$ wet weight) between individuals was validated.

The experimental diets were comprised solely of either fresh Ulva fasciata ('Ulva diet') or fresh Gracilaria conferta ('Gracilaria diet') grown in the local IMTA system, or a pelleted feed made of plant-meals that lack algae or any marine footprint but contain whole wheat, rapeseed, soya pulp, corn starch and other essential minerals and vitamins (Additional file 1: Table S1). These diets differ greatly in their polysaccharide content. In Gracilaria, galactans are the primary polysaccharides, classified as agarans and carrageenans, and the latter also consist of sulfate ester groups in their repeating disaccharides in the poly-chain [15]. In Ulva, sulfated ulvans are the main cell-wall polysaccharides and their content in dry biomass may reach $38-54 \%$ [16]. In contrast to these marine polysaccharides in the algal diets, the primary polysaccharides in the pelleted feed used are plant cellulases and starch. The feeding trial was set in a completely randomized block design with triplicate tanks for each treatment containing nine individuals each, all of which were nourished ad libitum for a period of eight weeks. Feeding ended forty-eight hours prior to examination of the sea urchins in order to minimize remnant digesta in the gut. The sea urchins' initial and final diameter, weight (dry and wet weight), and gonad weight, were measured in three randomly selected individuals in each tank. The 
specific growth rate and gonadosomatic index were then calculated as described elsewhere [12].

\section{DNA extraction and sequencing}

Three sea urchins from each tank (nine per diet type) were sacrificed for DNA sampling from the different gut regions. The tubular digestive tract was removed and separated into its three major regions [17] of the esophagus, which is closest to the mouth and where ingested food is covered by a mucous layer [18], and the anterior stomach and posterior intestine where secretion of digestive enzymes and nutrient absorption take place, respectively $[19,20]$. Dissection was performed using sterile instruments, while residual digesta was removed, and the remaining tissue was rinsed gently with Ultra-Pure water (Additional file 1: Fig. S1a-d). Gut samples were homogenized in FastPrep- $24^{\mathrm{TM}} 5 \mathrm{G}$ homogenizer (MP Biomedicals) and gDNA was extracted using the PureLink ${ }^{\mathrm{TM}}$ Microbiome DNA Purification Kit (Thermo Fisher Scientific, USA) following the manufacturer's protocol. Only DNA of high quality and quantity was amplified with the 515F (GTGYCAGCMGCCGCGGTAA) and 926R (CCG YCAATTYMTTTRAGTTT) primer set [21] by denaturation at $95{ }^{\circ} \mathrm{C}$ for $5 \mathrm{~min}, 28$ cycles at $94{ }^{\circ} \mathrm{C}$ for $45 \mathrm{~s}$, $50{ }^{\circ} \mathrm{C}$ for $60 \mathrm{~s}, 72{ }^{\circ} \mathrm{C}$ for $90 \mathrm{~s}$, and a final elongation at $72{ }^{\circ} \mathrm{C}$ for $10 \mathrm{~min}$. Bacterial $16 \mathrm{~S}$ rRNA gene sequencing was performed at the Research Resources Center (University of Illinois, Chicago) by the Illumina MiSeq platform [22] using the reagent kit v3. Quality and quantity assurance included examination of negative and positive control samples (using fecal samples as positive control) on which all procedures both before and after sequencing were performed.

\section{Data analysis}

Analyses of GMA were performed on 15, 18, and 21 samples from urchins fed with diets of Ulva, algae-free pellets, and Gracilaria, respectively. Different gut regions were represented equally in each sample set. Bioinformatics analysis was performed using the Quantitative Insights into Microbial Ecology pipeline (QIIME; version1.9.1) [23]. After removing primers and linkers, pairend reads were merged using PEAR [24], and sequences with a quality score below 30 or shorter than $300 \mathrm{bp}$ were filtered out. Sequences were clustered into operational taxonomic units (OTUs) at $97 \%$ similarity cutoff using UCLUST [25] and the Silva database (ver. 132) [26], and chimera sequences were removed by ChimeraSlayer [27]. A subsequent alignment step by PyNAST [28] identified master sequences according to the most abundant criteria. Taxonomy assignment was established using the UCLUST classifier while discarding OTUs represented by less than five sequences or those identified as chloroplast or mitochondria. A Linear discriminant analysis Effect Size (LEfSe) [29] at false discovery rate (FDR) $P<0.01$ was performed to identify bacterial taxa that can be considered as biomarkers of a certain diet or gut region.

Each of the microbes in the assembly was examined separately to determine whether it falls under the definition of core or unique microbe and secondly as an either generalist or specialist microbe. Core microbes were identified as those that appeared in at least $85 \%$ of samples of any specific habitat within the examined variable of diet, gut region, or both. On the other end, unique microbes were those that appeared in at least $85 \%$ of the samples of a specific gut region or diet but in no more than $25 \%$ of the samples of each of the other two diets or gut regions. Following this classification, niche breadth of each of the microbes was measured as recommended [30] by ranking OTU evenness of occurrence across various habitats (Shannon-Weaver diversity) [31], occupancy across habitats (Richness) [32], and the Levin's index [33]. Microbes with an overall rank of niche breadth within the top or bottom decile were defined as generalists or specialists, respectively, while all other microbes were defined as non-significant. Microbial association networks were analyzed using MetagenoNets [34] and the latent variable model Correlation interference for Compositional data through Lasso (CCLasso) [35] after log ratio transformation and measurement of edge weight (significant level of $P<0.005$ and permutation of 200) for each node pair, excluding OTUs with low prevalence $(<0.001 \%)$ and occurrence $(<10 \%)$. Topology indices of density, diameter, cluster coefficient, average path length, and Jaccard edge index were analyzed for networks and participating nodes by Cytoscape [36]. The randomization level of association networks was evaluated following the common distribution model of nodes' number of degrees [37]. Following the model, a random network will reveal Poisson distribution with many of the nodes having about the same number of degrees, while in a scalefree network a power-tail distribution is formed by a high number of nodes that have few degrees and vice versa. Hub nodes were identified using CentiServer [38] following their high degrees (edges), proximity centrality (short distance to any other node), between-ness (fraction of cases when node is in-between shortest track of all other pair nodes) and the Kleinberg's hub centrality [39] score as recommended $[7,40]$. In addition to hub taxa, driver nodes are defined as those leading to rewiring of the association network under a specific treatment. Therefore, they present a low proportion of shared edges with other networks (Jaccard edge index; JEI), as well as exclusive enrichment of interacting partners (neighbor shift score; $\mathrm{NESH}$ ) and a greater centrality (i.e., greater occurrence 
frequency in paths of other nodes) as compared to other nets $(\Delta B)$. Such nodes were extracted from data using NetShift [8] employing a combined threshold of JEI $<0.3$, $\mathrm{NESH}>1.8$, and a non-zero $\Delta \mathrm{B}$ which was validated via pair-wised tests against each of the other two networks (diets or gut regions). Since identification was carried out in sets of paired networks, it should be noted that driver nodes identified here achieved the driver criteria in one specific niche network when compared in pair to each of the other two networks. Moreover, such microbes failed the criteria when compared with the other two network pairs.

A further examination of the predicted metabolic and functional roles of the key bacterial taxa identified in T. gratilla elatensis was performed. To do this, the sequences of OTUs that were identified as key hub microbes and those categorized as core, core-generalists, generalists, specialists, or unique in a specific niche, were uploaded to the NCBI database for blasting with megablast [41]. Bacterial strains with the highest number of hits and an available representative genome were selected for further analysis of their metabolic repertoire. Considering the differences in the biochemical content of the experimental diets, the genomes of these bacteria were analyzed in the Carbohydrate-Active enZYmes database (CAZy) using the dbCAN2 meta server [42] to identify annotated genes that encode carbohydrate-active enzymes (CAZymes) of carbohydrate binding, carbohydrate esterases, glycoside hydrolases, and glycoside transferases. A heat-map of these results was produced to visualize the variation in the content and number of copies of the glycoside hydrolase $(\mathrm{GH})$ and polysaccharide lyase (PL) genes (enzyme classes EC 3.2.1.-, and 4.2.2.-, respectively) between the selected microbes.

\section{Statistical analyses}

MicrobiomeAnalyst [43, 44] was used for examining each diet or gut region separately and also the combined effects of both these variables together. Rarefaction of data at a sequencing depth of 10,000 reads resulted in 51 samples for which differences in ecological indices of richness, alpha and beta diversity were measured by Kruskal-Wallis and post hoc Dunn's multiple comparison test. Differences in microbial community composition were measured by Bray-Curtis dissimilarity and Permutational multivariate analysis of variance (PERMANOVA). Differences in relative abundance were verified by Kruskal-Wallis, at a false discovery rate (FDR) of $P<0.01$. We selected the non-parametric Kruskal-Wallis test after refuting the null hypothesis and confirming that data in the sequencing results are not normally distributed following a Shapiro-Wilk test of distribution of the residuals $(P<0.0001)$.

\section{Results and discussion}

\section{Diet shapes the gut microbe assembly of T. gratilla elatensis}

No mortality or morbidity of sea urchins was observed during the entire period of the feeding trial. Sea urchin individuals fed the Ulva diet had a growth rate of $0.33 \%$ $\mathrm{d}^{-1}$ as compared to 0.27 or $0.1 \% \mathrm{~d}^{-1}$ on diets of Graciaria or pellets $(P<0.05)$, respectively (Additional file 1 : Table S2). The faster growth rate under dietary Ulva as compared to Gracilaria or pellet diet is consistent with a previous study of $T$. gratila elatensis when fed similar diets of Ulva, Gracilaria and pellets over a much longer culture period of 400 days [12].

The fifty-one samples that reached sequencing depth of 10,000 reads resulted in 988,513 high-quality reads $(18,305 \pm 5208$ per sample) that were clustered into 434 unique OTUs $(117 \pm 48$ per sample; Additional file 1: Table S3). Neither diet nor gut region alone affected the richness of the GMA $\left(\mathrm{H}_{50,9}=15.37 ; P>0.05\right)$. The richest assembly was identified in the esophagus of the Gracilaria-fed sea urchins and the poorest assembly was found in the stomach of the pellet-fed sea urchins (Additional file 1: Fig. S2). The more diverse community in the anterior esophagus $\left(\mathrm{H}_{50,3}=6.483 ; P<0.05\right.$; Fig. 1a, b) agrees with results reported in previous studies on the sea urchin Lytechinus variegatus either prior to or after capture and rearing on artificial diet $[45,46]$. This may be attributed to the rapid interactions with the external water environment and short retention time of ingested feed in the esophagus [47]. Lower $\mathrm{pH}$ and oxygen and high secretion of digestive enzymes in the stomach [19, 48] may have limited the assembly diversity in this niche. A similar characteristic was noted in those fed with artificial pellets (Fig. 1c), perhaps indicating disruption of the assembly due to the lack of algae or any other marine footprint in this diet. Diet type restriction induced dissimilarity between gut assemblies $\left(\mathrm{F}_{50,3}=8.2, P<0.01\right.$, $R^{2}=0.25$, Stress $=0.143$; Fig. $\left.1 d, e\right)$. The effect was intense enough to also differentiate assemblies of the different gut regions $\left(\mathrm{F}_{50,9}=6.16, P<0.01, \mathrm{R}^{2}=0.539\right.$; Fig. $1 \mathrm{f}$; Additional file 1: Fig. S3a-c), with the strongest impact seen in the Gracilaria-fed sea urchins $(P<0.001$ Additional file 1: Fig. S3a). This combined effect of the diet and gut region variables together (as compared to the effect of only diet or only gut region) resulted in a more diverse assembly in the anterior esophagus as compared to posterior regions in Gracilaria-fed urchins $(P<0.01)$, while an exact opposite pattern was noted in Ulva-fed sea urchins $(P<0.02)$ (Fig. 1c). An interesting finding was the relatively low variation in Shannon index of diversity within samples from different sea urchins when fed the Ulva diet, in each of the examined gut regions of these sea urchins (Fig. 1c). This can be attributed to the relatively 


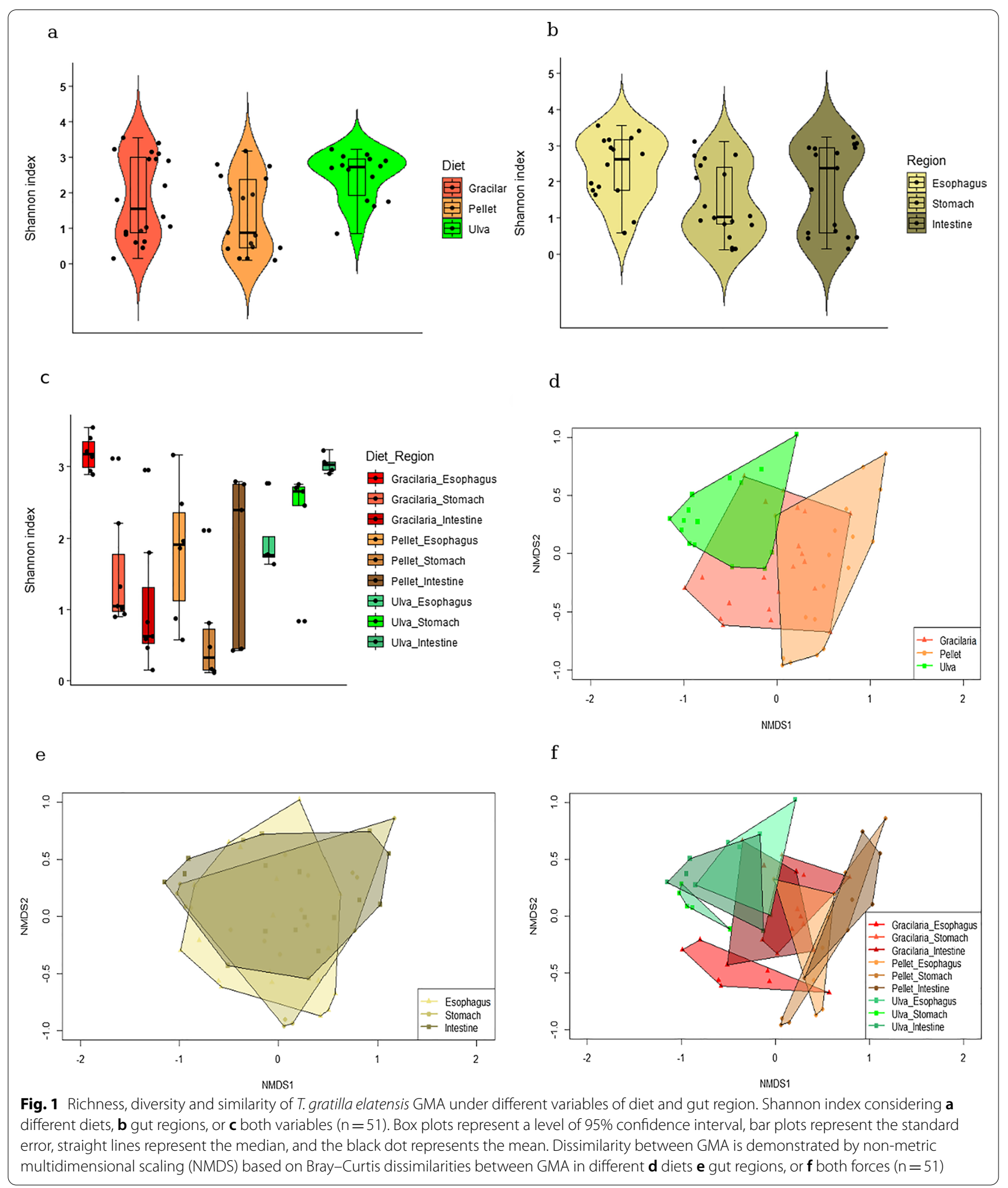

consistent prevalence of the dominant taxa in the Ulvafed sea urchins across samples of different biological replicates (sea urchin individuals; Additional file 1: Fig. $\mathrm{S} 4 \mathrm{~b})$, which also resulted in a relatively low variation 
within individuals in terms of the community composition and diversity.

\section{Predominance of bacterial taxa in the gut of T. gratilla elatensis}

None of the 110 genera (Fig. 2a) from 17 bacterial phyla (Additional file 1: Fig. S4a, Table S4) predominated in a particular gut region, but the three taxa, Cyanobacteria, Firmicutes, and Fusobacteria, were predominant in the assembly in Ulva-fed sea urchins (Fig. 2b, $P<0.001$ ) and exhibited (each in itself) a relatively similar prevalence in the different gut regions in all the examined diets. An opposite prevalence pattern of Spirochaetes vs. Tenericutes was evident under the Gracilaria diet. The a
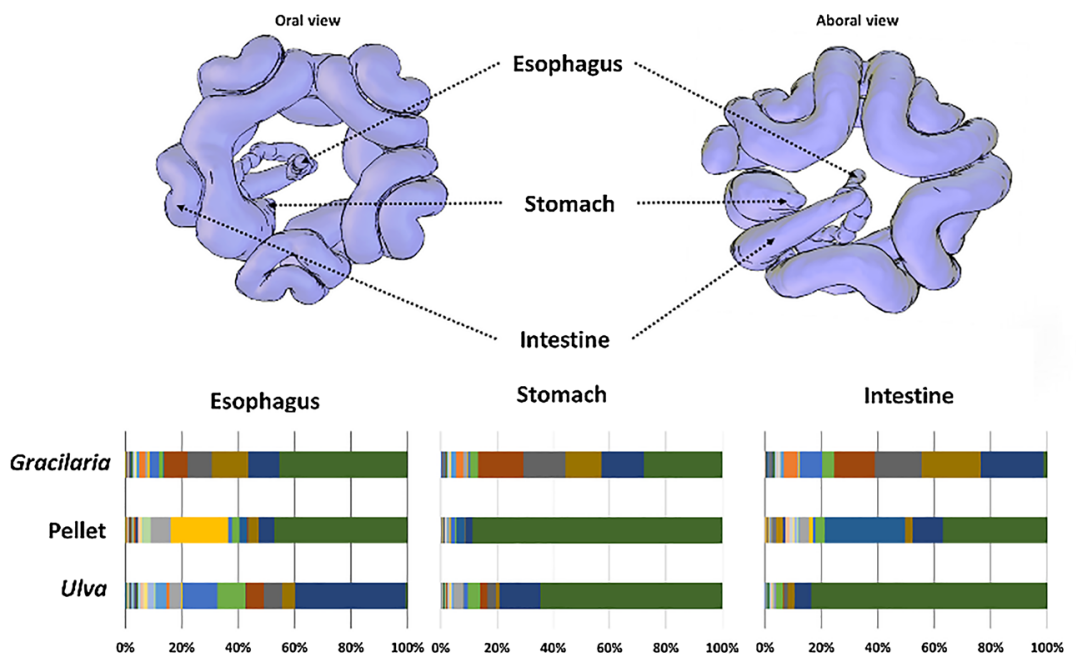

Relative abundance

\begin{tabular}{|c|}
\hline 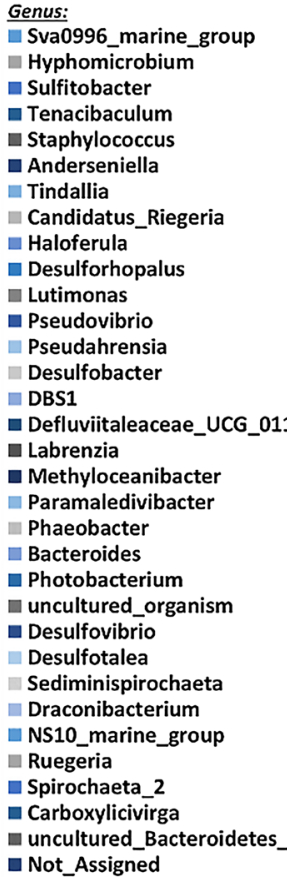 \\
\hline
\end{tabular}

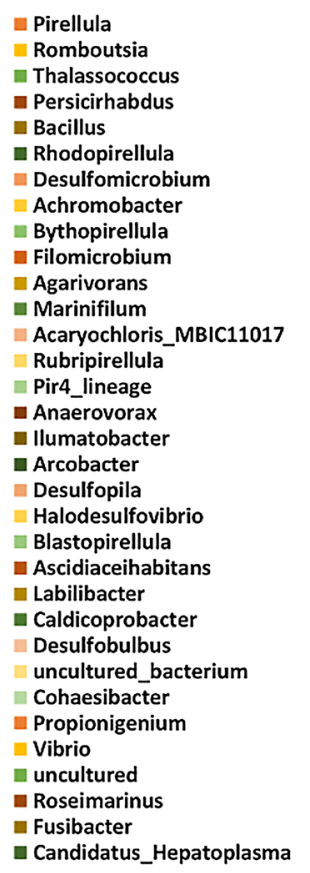

$\mathrm{b}$

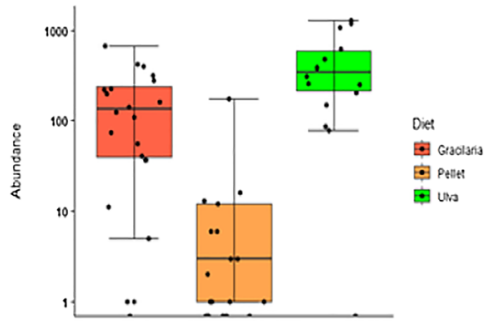

Cyanobacteria

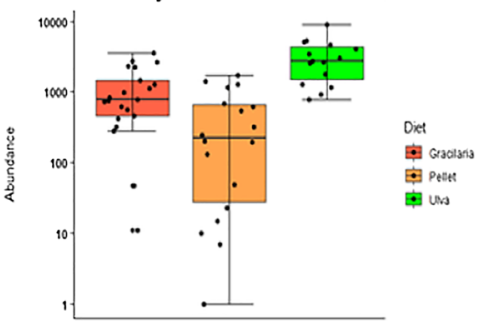

Firmicutes

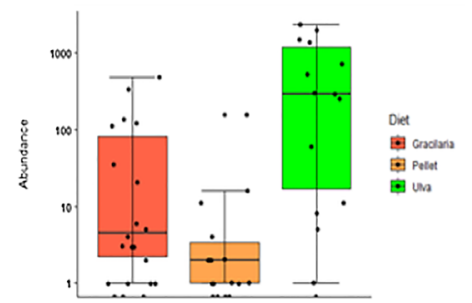

Fusobacteria

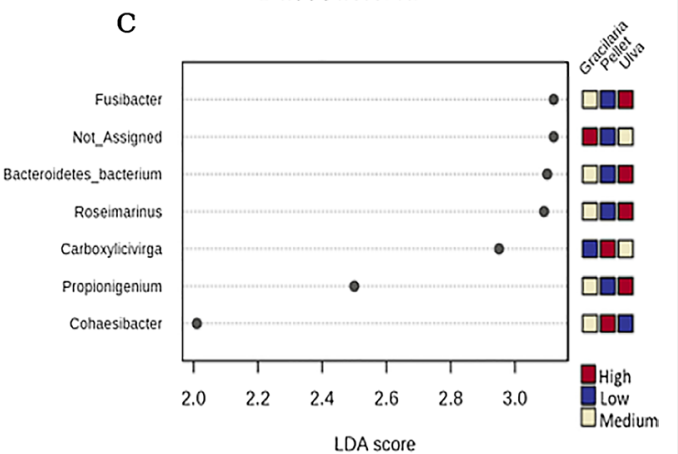

LDA score

Fig. 2 T. gratilla elatensis GMA composition under different variables of diet and gut region. a Illustration of sea urchin digestive tract (performed using magnetic resonance imaging [49] and image illustrator at https://www.nhm.ac.uk/our-science/data/echinoid-directory) followed by the relative abundance of T. gratilla elatensis gut microbes (Genera) in different gut regions and under each diet $(n=54)$. $\mathbf{b}$ Log-transformed count of bacterial phyla with greatest differences in prevalence under a particular diet, middle line represents median, and whiskers are drawn from the 10th to 90 th percentiles $(n=54)$. c Gut bacterial genera identified as diet-biomarkers in T. gratilla elatensis via Linear discriminant analysis $(n=54)$ 
Spirochaetes, primarily represented by the genus Spirochaeta, predominated in the esophagus and decreased in posterior regions, while the Tenericutes, which was primarily represented by a single OTU assigned as genus Candidatus hepatoplasma, predominated in the intestine and stomach and was lower in the esophagus $(P<0.001$; Additional file 1: Fig. S4b). Fluctuations in prevalence allowed identification of potential diet biomarkers in the GMA (Fig. 2c) as the genera Fusibacter, uncultured Bacteroidetes bacterium, Roseimarinus, and Propionigenium for Ulva; Carboxylicivirga and Cohaesibacter for pelleted feed; and unassigned for Gracilaria; but none were specific to any gut region.

\section{Niche specification in the gut microbial assembly of sea urchins}

Generalist and core microbes colonize the majority of examined ecological niches, i.e., the different gut regions of $T$. gratilla elatensis, fed on the different diets. Therefore, these microbes may be highly important in aiding their host in changing environments [50,51]. Adhering to the fixed rule of colonization of at least $85 \%$ of the examined samples (across any gut region and/or diet), the core microbes identified in the gut of T. gratilla elatensis included five OTUs belonging to taxa Candidatus Hepatoplasma, Ruegeria, Vibrio, and two uncultured members of the order Bacterioidia (uncultured bacterium and uncultured Bacteroidetes bacterium) (Table 1). Two additional taxa of uncultured Fusibacter and Roseimarinus were thusly determined as core microbes in all the examined gut regions and under diets of Ulva and Gracilaria. However, they marginally failed the selection threshold criteria in the pellet-fed urchins, appearing in only $83 \%$ of the samples under this diet. The cumulative abundance of each of the identified core microbes was above 10,000 reads (Fig. 3a). Moreover, the relative abundance of a core microbe obtained in any sample was greater than $0.01 \%$. These findings on the occurrence and abundance of core microbes may aid in their confirmation as resident microbes in the gut of $T$. gratilla elatensis rather than transient microbes which may only pass through this organ.

Identified generalists included 18 microbes ( 8 of them assigned to phylum Proteobacteria or class Planctomycetacia) with relatively high occurrence $(>54 \%)$, diversity (>2.95), and niche breadth (>13.38; Fig. 3a). The 12 identified specialists were mostly annotated to Bacterioidetes (Table 1; Fig. 3a). Three core microbes of the respective genera of $C$. hepatoplasma, uncultured Bacteroidetes bacterium, and Fusibacter were also among generalists (i.e., core-generalists) and revealed the highest cumulative abundance (Fig. 3b). The highest occurrence and abundance of these core-generalists agrees with the predomination of these taxa in the GMA of other sea urchin species as well [52-54]. A recent model highlighted the important role of the generalist-specialist evolutionary cycle which allows the spreading of specialist species across diverse environments, thereby maintaining taxonomic diversity [2] while at the same time influencing the overall community and its differentiation [2, $3]$. Following the model, the generalist-specialist cycle includes the expansion of generalist microbes across various ecosystems, where local environmental forces determine the specialization of some of their descendants in the specific niche, hence the latter's determination as specialist microbes [2]. In another study, core microbes in fish gut revealed high strain variability [30].

Findings in the current study may suggest the fitness of phylum Bacteroidetes to this generalists-specialists cycle model with core and core-generalist OTUs (of genus of Roseimarinus and uncultured Bacteroidetes bacterium, respectively) as potential founders in the gut assembly of T. gratilla elatensis, and several other OTUs of these phyla (four of the genus Roseimarinus and one uncultured Bacteroidetes bacterium) identified as specialist microbes that occupied a narrower unique niche of the gut of Ulva-fed sea urchins. The fact that Bacteroidetes reveal high plasticity and genetic rearrangement, allowing their rapid adaptation to distinct ecological niches [55], makes them more adequately fit the suggested evolutionary model in the examined sea urchin. Despite the significant differences in niche breadth between generalists and unique or specialist microbes, the total abundance of microbes from these groups was relatively similar (Additional file 1: Fig. S5). This indicates that the prevalence of unique or specialist microbes surpassed that of generalists in each of the niches they occupied. This notwithstanding, the core microbes were present in the highest abundance in these niches.

Intriguingly, unique microbes were identified only in sea urchins fed with algae: Thirteen of these in Ulva-fed (annotated to phyla Bacterioidetes, Cyanobacteria, Firmicutes, Spirochetes, Tenericutes, and unassigned), two in Gracilaria-fed (Bacterioidetes and Planctomycetes), and one unassigned taxon that was shared between these diets (Table 1). One explanation for the Ulva- or Gracilaria-unique microbes identified here may be the previous identification of members of Bacteroidetes, Cyanobacteria, Firmicutes, Spirochaetes, and Plancomycetes taxa as epibionts on these algae $[56,57]$. However, the relatively high Shannon diversity and Levin's indices of many of these OTUs that were measured in sea urchins fed these diets may also be explained by high specialization of these microbes in the narrow diet-mediated niches. This is particularly evident in the sea urchinassociated Bacteroidales and Clostridales fed on an Ulva 


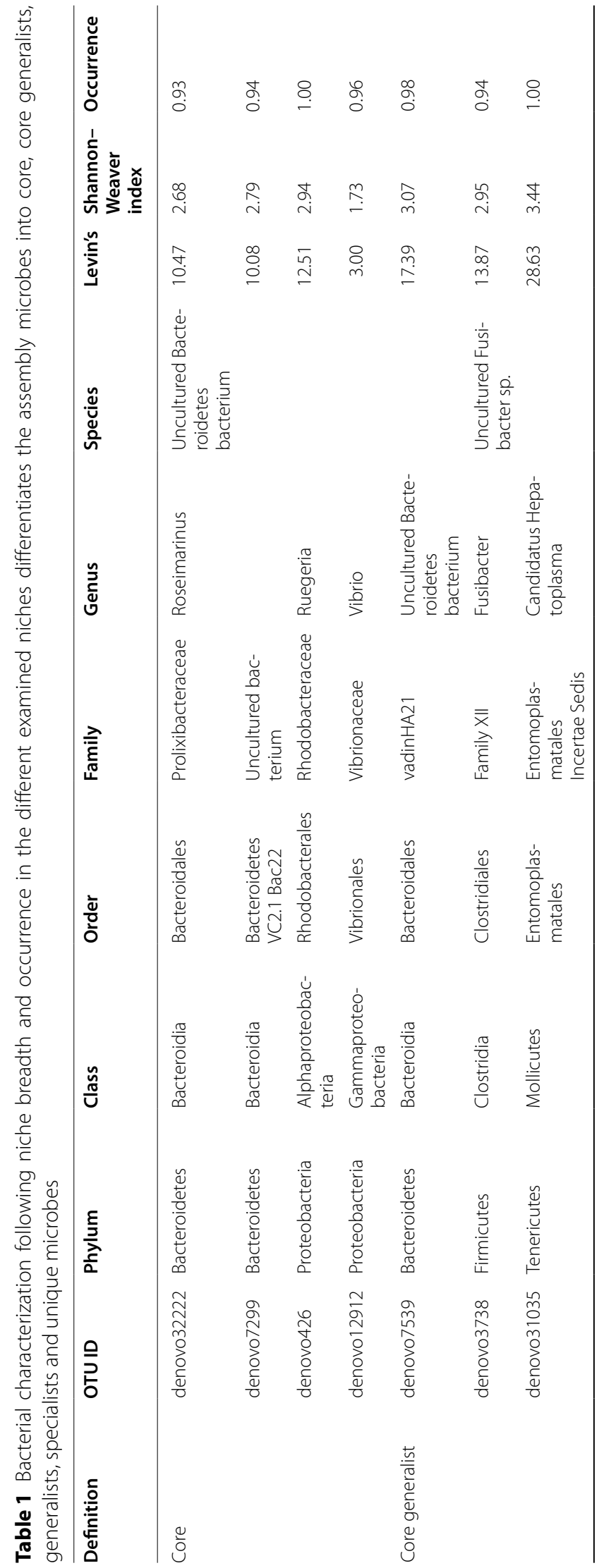




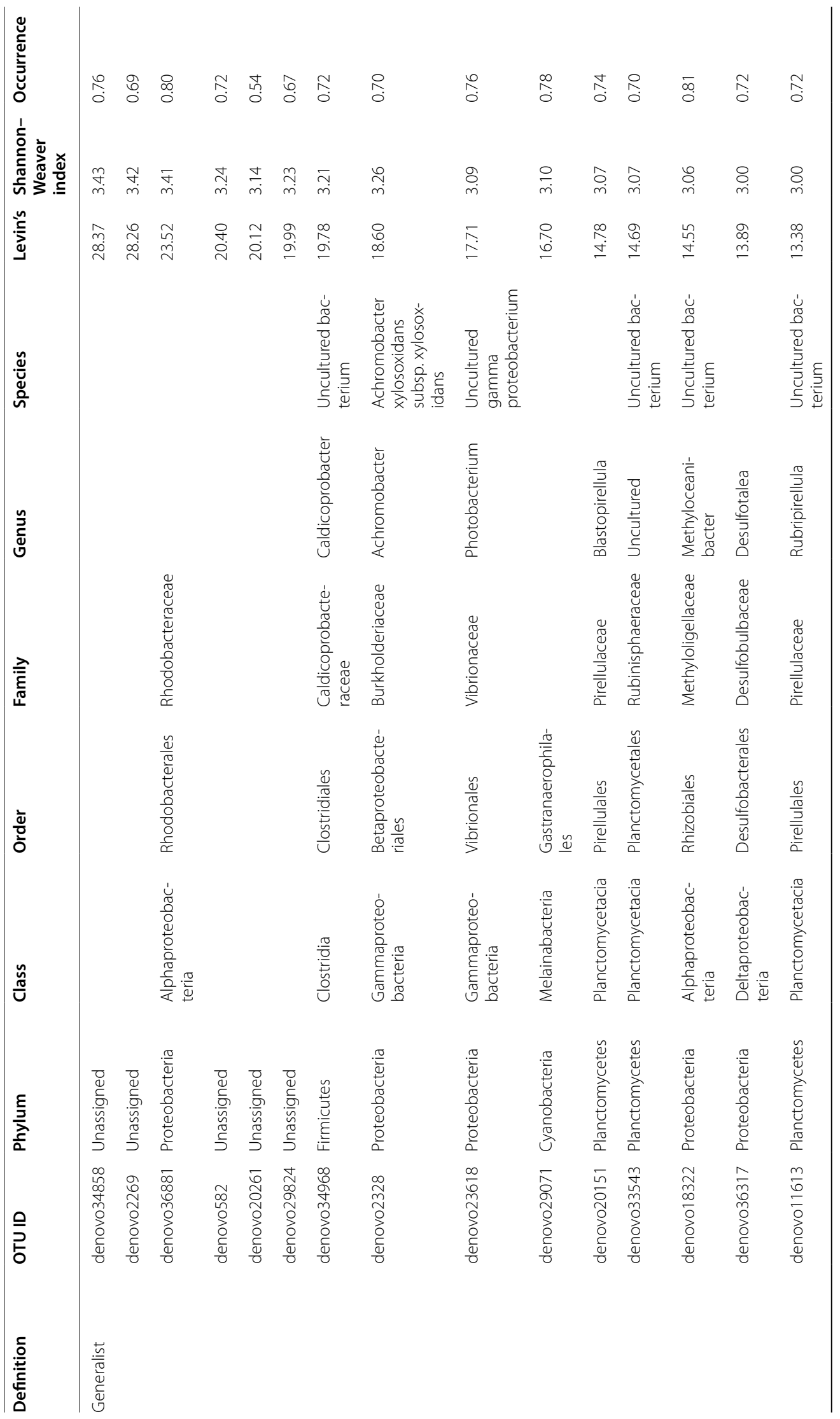




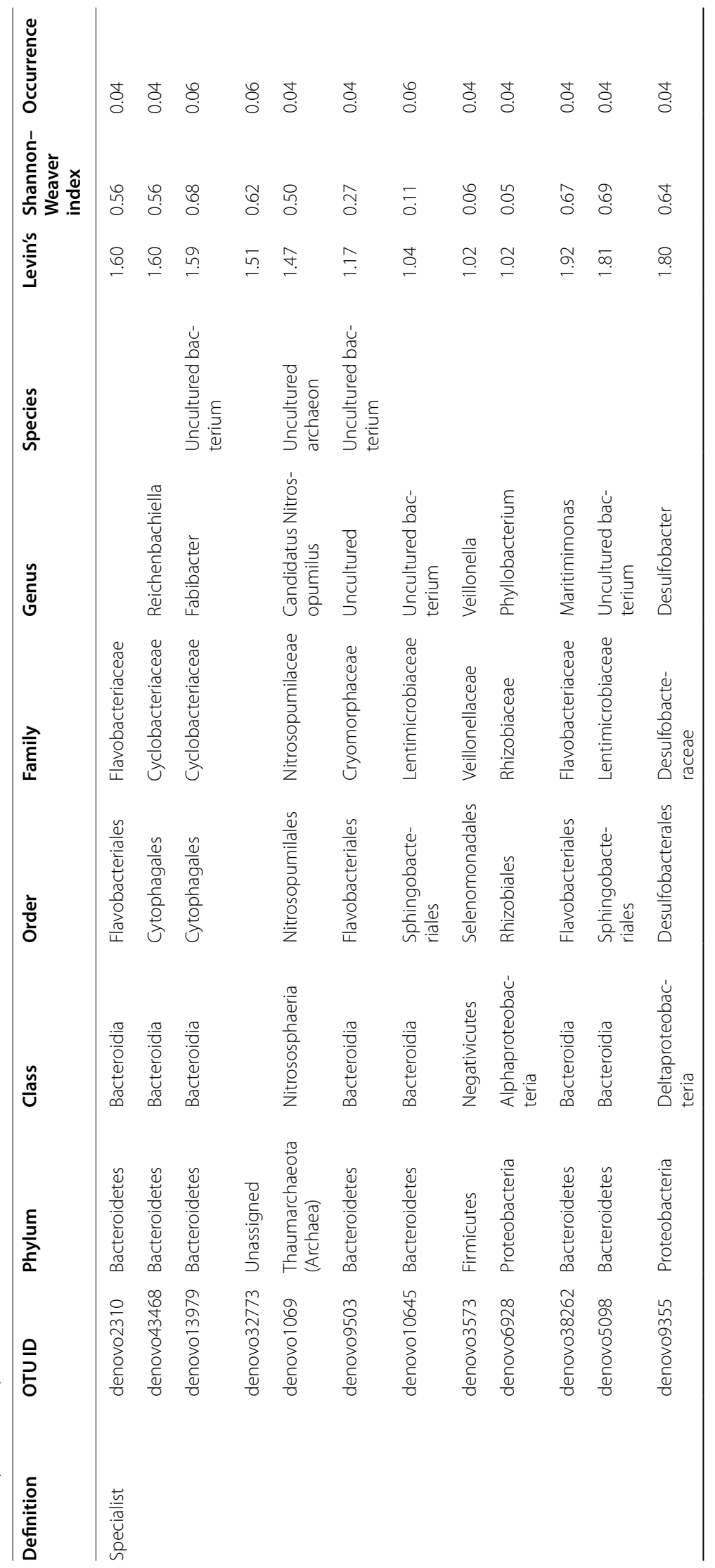




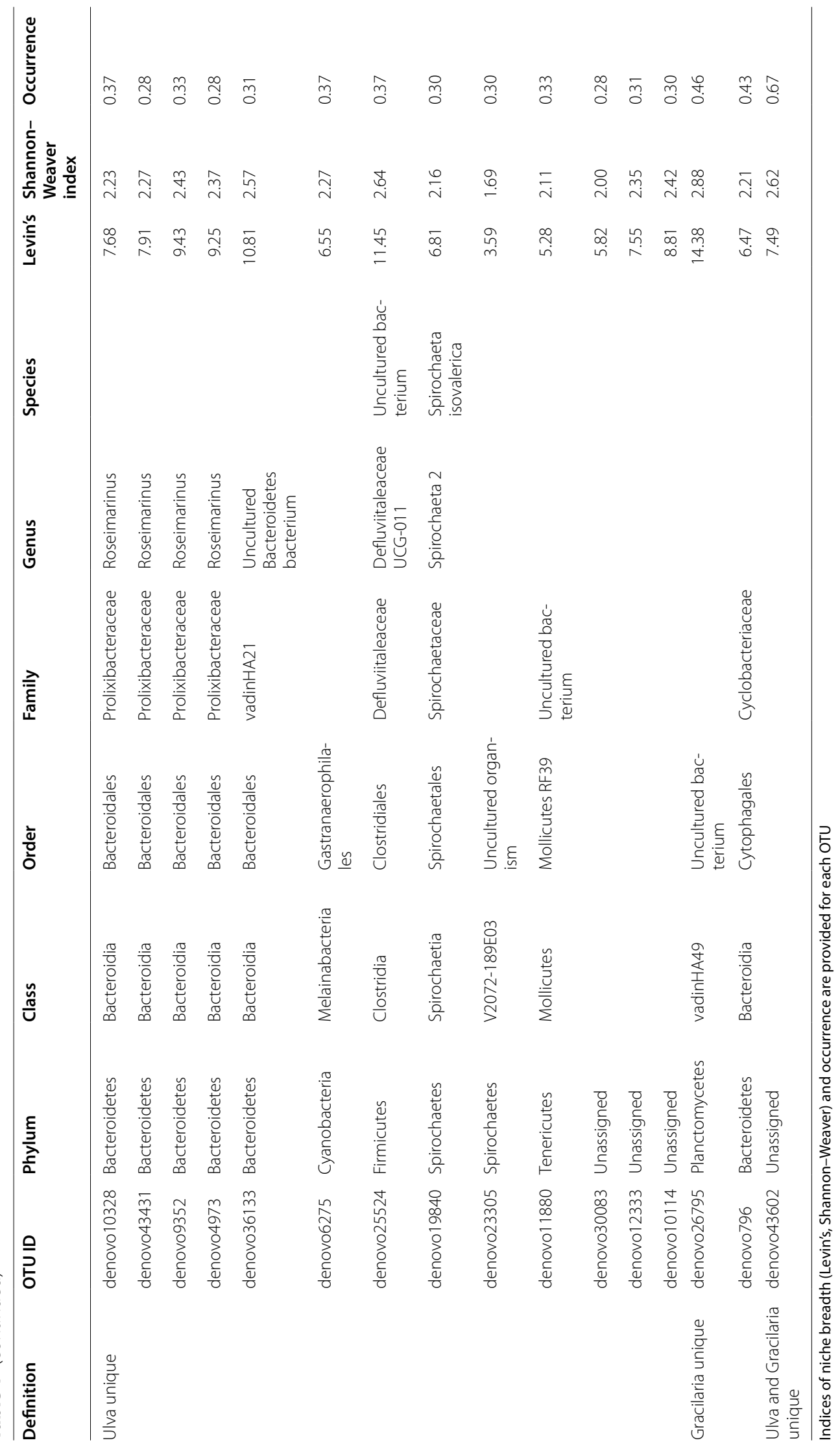


a

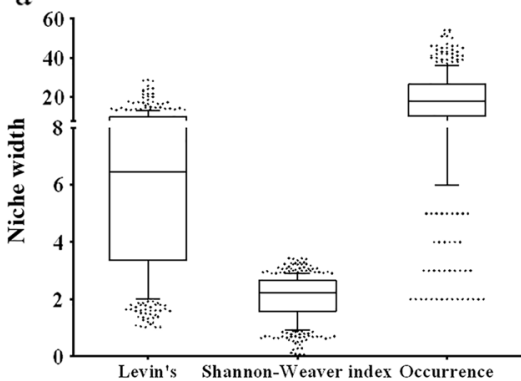

$\mathrm{b}$

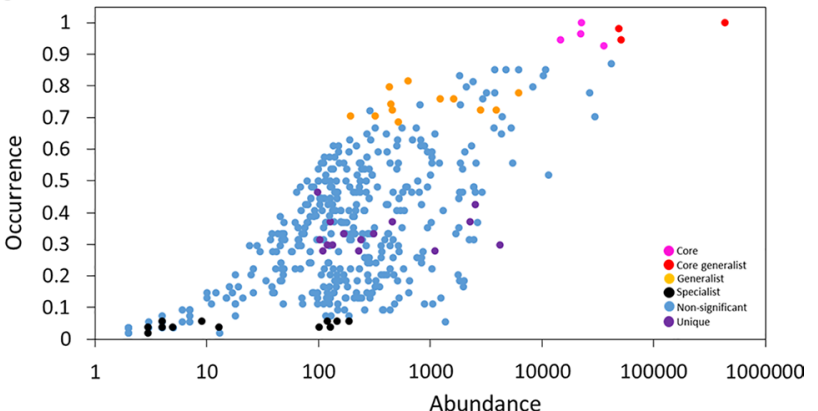

Fig. 3 a Niche breadth of microbes in sea urchin GMA following the different measured indices of Levin's, Shannon-Weaver, and occurrence. Middle line in box plots represents mean value and whiskers are drawn from the 10th to 90th percentiles. Generalist or specialist microbes are shown at top or lowest decile, respectively $(n=434)$. $\mathbf{b}$ Occurrence and cumulative abundance of each of the microbes in GMA indicates differences of niche breadth and specialization in unique habitats. Each dot represents one microbe with a color indication indicating core, core-generalist, generalist, specialist, unique, or not significant $(n=434)$

diet and in sea urchin-associated uncultured bacterium (Planctomycetes) fed on the Gracilaria diet. Correspondingly, the contribution of these microbes to the decomposition of the complex polysaccharides in Ulva or Gracilaria should be considered, particularly since, despite their algivorous life style, sea urchin digestive systems contain few algal polysaccharide digestive enzymes $[47,58]$.

\section{Assembly connectivity is determined via niche-specific hub and driver microbes}

The overall association network of the GMA consisted of 27 nodes (each representing a single OTU/microbe) with 37 edges (i.e., of either co-occurrence or co-exclusion; also referred to as degrees). This network, however, was separated topologically and contained three sub-nets, each containing many fewer clusters of the participating microbes (Fig. 4a). This low connectivity of the general network highlights the importance of investigating microbial networks in the narrower niches of each different diet or functional region (Fig. $4 \mathrm{~b}-\mathrm{g}$ ) to also allow the identification of niche-specific associations, clusters, hub microbes, or other changes. Examination of the variation in number of degrees between participating nodes in the different networks revealed a Poisson distribution of the degrees per node in networks of the different gut regions but a power-law tail distribution of this index in networks under different diets (Additional file 1: Fig.
S6). These results suggest that the microbial networks in different gut regions or under different diets can be differentiated into random or scale-free networks, respectively [59]. Notably, many nodes in the network of the Ulva-fed sea urchins possessed a high number of degrees and formed a left tail distribution shape. This was exceptional compared to the other two diets, where only a few nodes revealed a high number of degrees, i.e., right tail distribution (Additional file 1: Fig. S6a-g). Considering the nature of associations, i.e., positive co-occurrence or negative co-exclusion, the number of positive associations in all examined niches surpassed that of the negative ones, with the greatest fraction of the total edges in the Ulva and intestine networks. However, the nature of many of the shared associations between networks was of competition/co-exclusion between Cyanobacteria and Bacteroidetes or Firmicutes (diets), or between nodes of Cyanobacteria, Spirochaetes, Firmicutes, Tenericutes, and Bacteroideteds (gut regions) (Additional file 1: Table S5).

Examination of the topology indices of the co-association networks (Additional file 1: Fig. S7a-g) revealed similarity in terms of the number of edges between participating nodes in networks of the different gut regions (14-16\% similarity), but under different diets the Ulva network revealed a greater dissimilarity from any other diet network with $\mathrm{ca}$ 2.5-fold more edges (Additional file 1: Fig. S7b). The significant high edges number in the Ulva network may indicate cross feeding relationships

(See figure on next page.)

Fig. 4 Association network of T. gratilla elatensis GMA under different variables of diet and gut region. Association network presenting a the general network (regardless of diet or region), and in different examined niches of diets b Gracilaria, c Pellet, or d Ulva; or gut regions e esophagus, $\mathbf{f}$ stomach, or $\mathbf{g}$ intestine. Diamonds indicate hub nodes and triangles indicate driver nodes. Nodes are colored following annotation at Phyla level. Edge color indicates type of association as either co-occurrence (blue) or co-exclusion (red). $\mathbf{h}$ Venn diagrams reveal number of shared or unique associations in networks of different diets or gut regions $(n=54)$ 


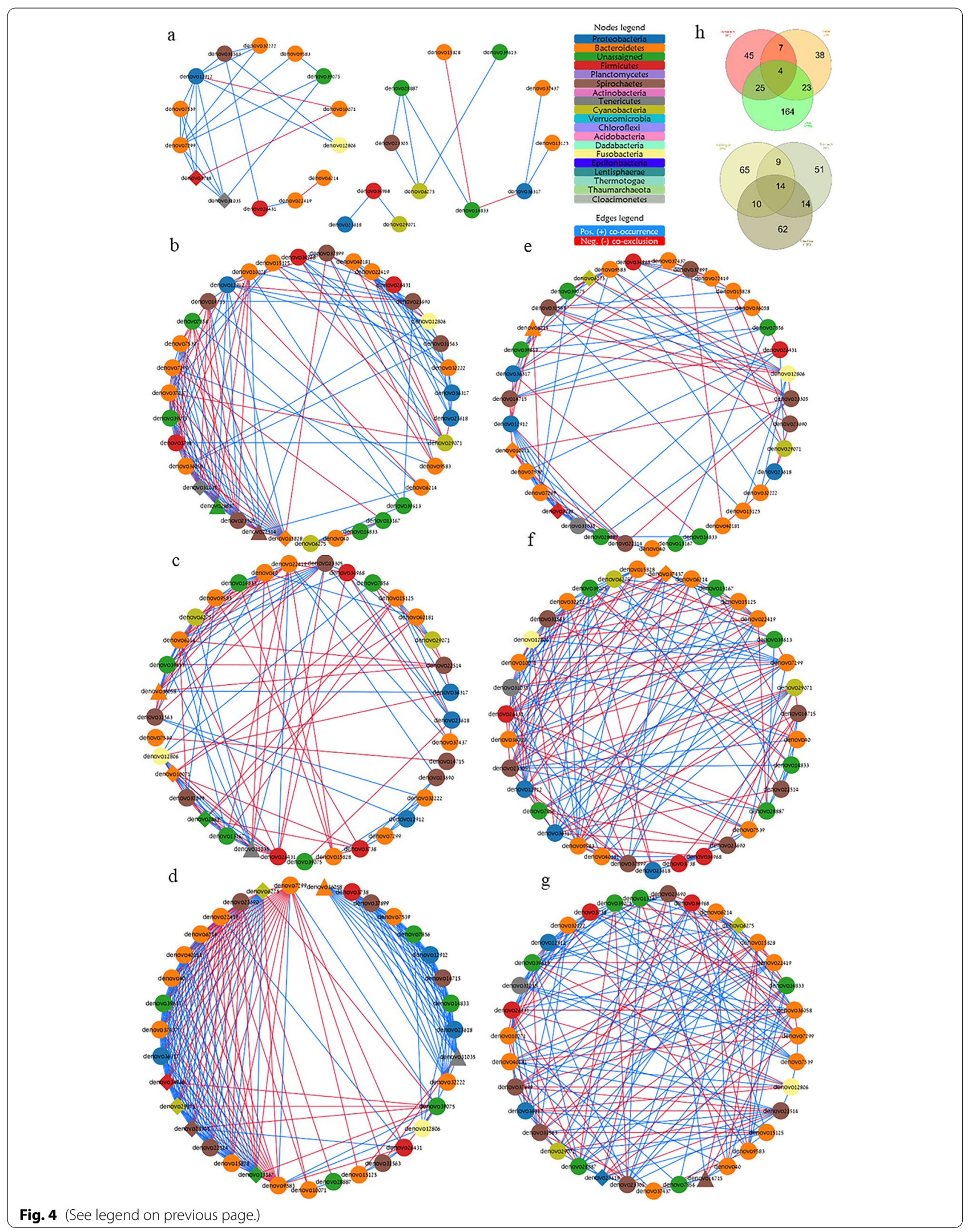


and richness in feed decomposition pathways [60]. The high number of edges under the Ulva diet resulted in many unique edges, $76 \%$ of total edges (Fig. 6h), but also in a significantly denser network and more sub-clusters of participating nodes than were seen in other diets (Additional file 1: Fig. S7d,e). Therefore, it is more likely that under the Ulva diet, any two microbes in the assembly will perform a direct association between themselves (most likely a positive one), as both the diameter and average path length were similar to those measured in other networks under different diets [61].

Our finding of a contradictory high-tail degree distribution under Ulva diet compared to other diets supports the advantage of the multi-parametric analysis for hub node identification as performed here. This analysis enabled the identification of an unassigned node as a hub microbe in the Ulva network as per its significant "high betweenness centrality" despite the somewhat lower "number of degrees". The hub in the different networks included OTUs from six phyla of Bacteroidetes (3 OTUs of order Bacteroidales), Firmicutes (2 OTUs of order Clostridiales), Cyanobacteria (2 OTUs of order Gastranaerophilales), Proteobacteria (1 OTU of genus Photobacterium), Spirochaetes (1 OTU), Tenericutes (1 OTU of genus Candidatus hepatoplasma) and two more unassigned OTUs (Additional file 1: Table S6). Our identification of the hub nodes in the different networks as per the criteria for hub microbes [7] suggests Phylum Bacteroidetes as the keystone taxa, with a representative hub OTU in nearly all examined networks (Additional file 1: Table S6). Our additional findings of the relatively high strain variability of Bacteroidetes (richness; a total of 111 OTUs, $2^{\text {nd }}$ highest) in the gut of $T$. gratilla elatensis sea urchin suggest further support for the importance of this keystone phylum. This strain variability revealed 3 core microbes, 7 specialist microbes (assigned to 5 different orders), and 4 Ulva-unique microbes (all assigned to genus Roseimarinus), all of this phylum.

Interestingly, the identified hub nodes in each network of the algal diets formed co-occurrence subclusters. Such a sub-cluster was also identified in the pellet-fed group but it included a fair number of coexclusions between the hub nodes (Additional file 1: Fig. S8). Considering these findings, the synchronization between hub nodes in networks of the algal diets in terms of their co-occurrence may suggest a major role of these microbes in syntrophy/cross-feeding via complementary metabolisms [62]. Such syntrophy is greatly influenced by available resources (including feed) [63] and allows maintenance of functional redundancy in the community due to the presence of multiple taxa with shared metabolic function even when a particular node is eliminated from the network, $[60,64]$. Under the algal diets tested here, this microbial syntrophy may be essential for the metabolism of the complex fibers that are the primary ingredients in these feeds $[65,66]$. This could be compared, perhaps, with the syntrophic microbial metabolism of cellulases in cows' rumens [67]. This is further supported by the fact that microbe connectivity in different regions of the gut was mainly through in-line/chain-form connections despite being spatially close in each of the examined regions.

Transfer of the sea urchins from the initial baseline algal diet of combined $U$. fasciata and G. conferta to the pellet diet treatment may have caused a more severe disturbance of their GMA than did the other two diet treatments in which the change was simply restriction to only one of these two algal species. This disturbance may have resulted in reduced diversity and a less dense network, consisting of low numbers of edges (also of exclusive ones), a fair number of co-exclusion associations, and fewer sub-clusters (Fig. 4b-d,h, Additional file 1: Fig. S7b,d,e). This perhaps indicates reduced stability and functionality of the bacterial community $[6,68]$ which may have resulted from switching the urchins to this new artificial diet made of ground-up land crops rather than natural marine feeds. A similar reduction was also measured in rodents when they were transferred to and from their natural feed as a result of periodic captivity [69], and in captive fish after rearing on an artificial pelleted feed [70].

Network rewiring was greatest due to dietary influence, particularly between the pelleted and either of the algal diets, and less significant regarding the gut regions (Additional file 1: Fig. S9a-f). This discrepancy was also supported by the relatively high number of driver microbes that rewired diet-type networks as compared to their numbers in gut-region networks (Additional file 1: Table S7). Six microbes were identified as clear driver nodes due to their altered associations and

(See figure on next page.)

Fig. 5 Driver nodes to rewire T. gratilla elatensis GMA association network under different variables of diet and gut region. Driver microbes were extracted from networks using NetShift following significant change in degrees and associated partners. Only nodes that presented significant change as compared to the other two diets or regions were identified as driver microbes. Associations of driver nodes in $\mathbf{a}$ different diets or $\mathbf{b}$ gut regions. Annotation (genus level) of driver microbes include (upper to lower order): denovo22514 (Spirochaeta2), and denovo28887 (Unassigned) as driver microbes in the Gracilaria-diet network; denovo36058 (Roseimarinus) in the pelleted-diet network; denovo31035 (Candidatus Hepatoplasma) in the Ulva-diet network; denovo6214 (Roseimarinus) in the esophagus network; and denovo14715 (uncultured bacterium of Spirochaetes) in the intestine network. Nodes are colored following annotation at Phyla level $(n=54)$ 


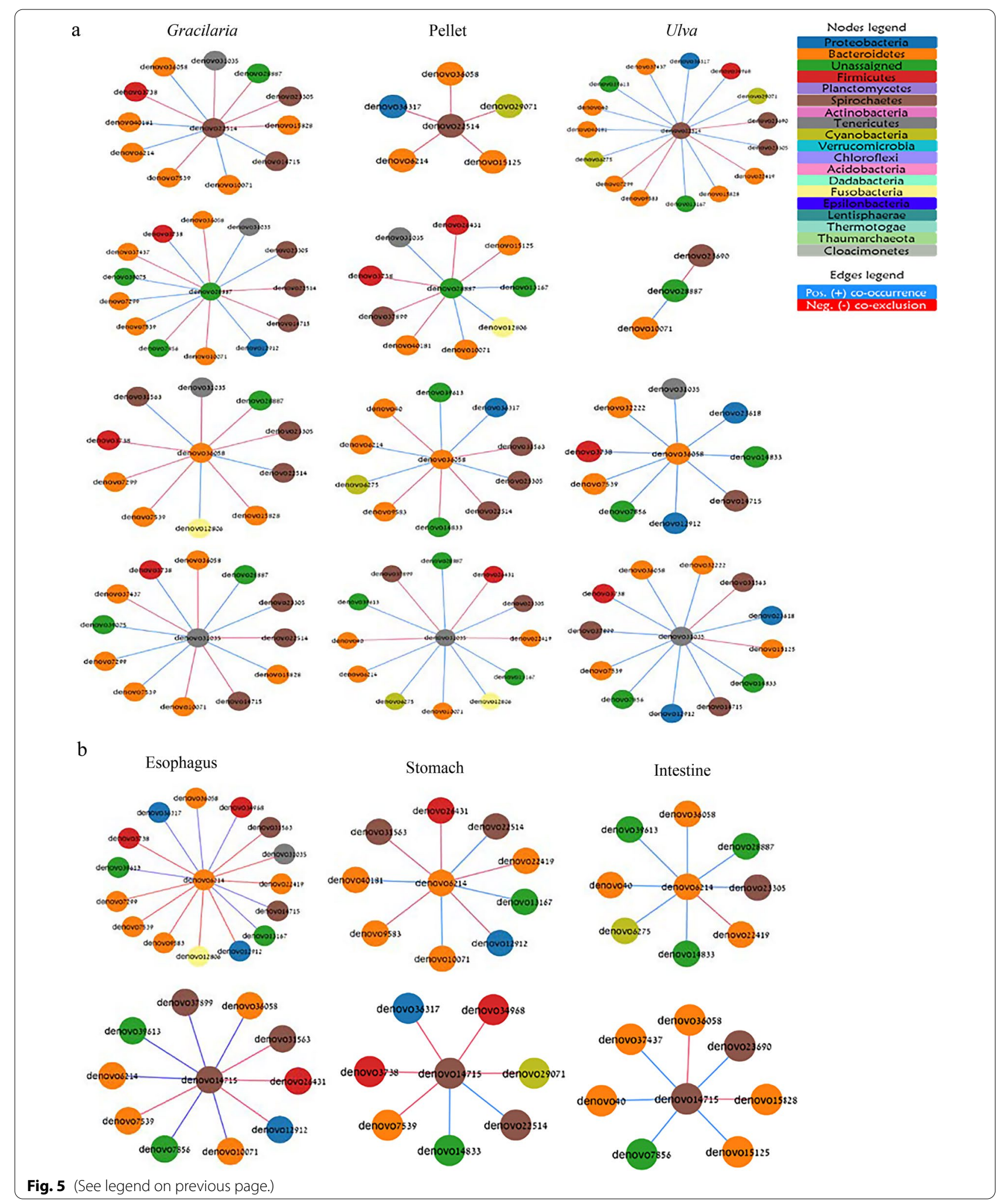




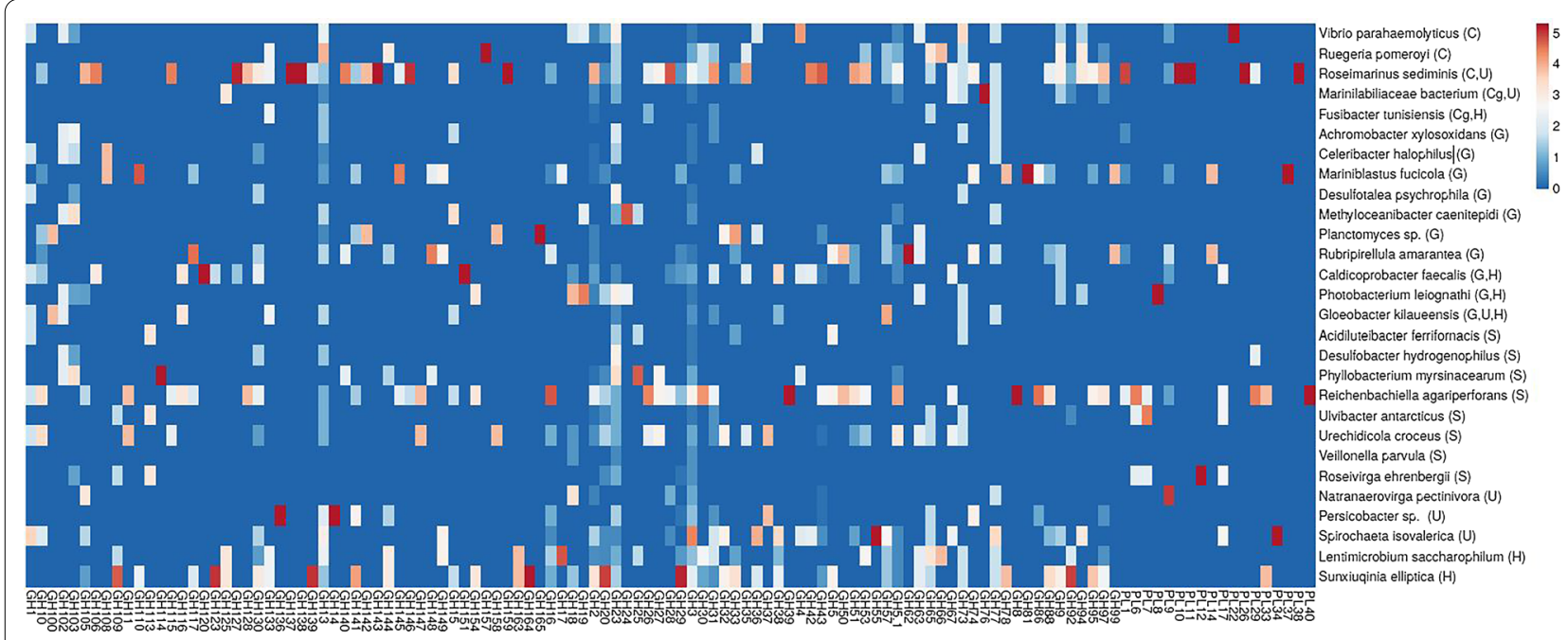

Fig. 6 A heatmap diagram of the variation in content and number of copies of glycoside hydrolase (EC 3.2.1.-) and polysaccharide lyase (EC 4.2.2.-) genes in the genome of the identified key microbes that fell into the determination of core (C), core-generalist (Cg), generalist (G), specialist (S), or unique $(U)$ microbes, and the keystone hub microbes in the microbial networks $(H)$

higher centrality in only one particular niche (Fig. 5). The networks of the pelleted diet and esophagus were rewired by differing driver nodes of Roseimarinus, while driver nodes of Sediminispirochaeta or C. hepatoplasma rewired the intestine or Ulva network, respectively. The lack of associations of Ulva-driver node C. hepatoplasma with hub nodes in this network was significant as compared to other drivers found in other networks. Therefore, despite the highest occurrence and abundance of this single OTU of phylum Tenericutes in all niches and examined sea urchin individuals, we assume that $C$. hepatoplasma acts as a host-dependent node in the assembly, as was also suggested for this bacterial species in the case of another sea urchin [46]. Under the Gracilaria diet, network rewiring was driven by two nodes, one of Spirochaetes and another of an unassigned microbe. These two drivers revealed a competitive association with each other under this diet. Moreover, while the Spichochaetes driver revealed negative interaction with all hub nodes of Gracilaria network, the other driver node (unassigned) co-occurred with them. As compared to other drivers, the unassigned driver in Gracilaria network also revealed greatest exclusive edges, i.e., interaction with an exclusive partner or with the same partner but via opposite association. Although both hub and driver nodes reveal great centrality in the network, the high number of degrees characterized the former but not necessarily the latter. However, in some cases where the node creates exclusive degrees that also increase its overall connectivity, the microbe is identified as both hub and driver node in this niche. This was the case with the unassigned and
Roseimarinus nodes in Gracilaria and esophagus networks, respectively (Fig. 5, Additional file 1: Table S6, S7).

\section{Generalism and specialism with regard to the potential metabolic functions of key microbes in sea urchin gut}

A primary concern in the current examination of the niche breadth, specialization, and networking of the microbes in T. gratilla elatensis gut was the validation of these results in terms of metabolic diversity. This has been highlighted as a key milestone that will improve our current understanding of the customary ecological definitions of generalism and specialism [51, 71, 72]. To do this, analysis was made of the potential metabolic repertoire in genomes of the key microbes identified in the gut, including the key hub, core, core-generalist, generalists, specialists, and the unique microbes, particularly concerning their richness in CAZymes. Some specific differences between the selected microbes were observed (Fig. 6, Additional file 1: Table S8) which can be attributed to a specific gut region or diet type, and in some cases also to a specific stage in the metabolism of dietary polysaccharides. Altogether, the genomes of the selected microbes presented a total of 271 carbohydraterelated enzyme classes (including sub-families) in the CAZy database. 167 of them were classified as glycoside hydrolases (GHs), 27 as polysaccharide lyases (PLs), 36 as glycoside transferase (GTs), 23 as carbohydrate binding modules, 12 as carbohydrate esterases (CEs), and 6 related to auxiliary activities. Of these classes, only GHs and PLs are involved in polysaccharide decomposition [73] and were further analyzed. Three bacterial strains of 
Roseimarinus sediminis, Reichenbachiella agariperforans, and Sunxiuqinia elliptica (all members of the phylum Bacteroidetes and isolated from marine environments) were richest in terms of the number of different CAZyme classes. These species were also richest in their content of CAZymes for polysaccharide decomposition, with 81 (R. sediminis) 73 (R. agariperforans), and 50 (S. elliptica) GHs and PLs. Examination of the genome of $R$. sediminis revealed 4 exclusive PL classes $(10,11,29$, and 38), while among these classes both the PL29 and PL38 (characterized as chondroitin-sulfate-ABC endolyase and glucuronan lyase, respectively) presented the highest number of copies in the genome. These findings appear adequate for the correlation of the four OTUs which were unique to Ulva-fed sea urchins in the current research (i.e., Ulva-unique) and annotated as $R$. sediminis, to potential activity in the metabolism of Ulva-polysaccharides in $T$. gratilla elatensis gut. The rich content of CAZymes in the genome of S. elliptica agrees with the known capability of this fermentative microbe to decompose various carbohydrates such as D-fructose, L-rhamnose, D-xylose, myoinositol, sorbitol, trehalose, D-mannitol, ribose, raffinose, gluconate, malonate, and propionate, each as a sole carbon source [74]. Hence, this characteristic may be attributed to our current finding of $R$. sediminis as a key hub node in the stomach of T. gratilla elatensis, where rapid fermentation is required for the metabolism of refractile and sulphated carbohydrates $[48,75,76]$ such as those derived from decomposition of the complex polysaccharides in Ulva and Gracilaria. We also suggest additional members of Bacteroidetes identified here as correlated with the initial depolymerization stage in the metabolism of Ulva or Gracilaria to be key taxa in the gut of sea urchins fed these diets. Among these is R. agariperforans which consists both of ulvan lyases (PL40 and PL37) and $\beta$-agarases (GH50 and GH86) that are correlated with degradation of Ulva-ulvan [77] and Gracilaria-agarose [78], respectively. The $\beta$-agarase GH86 is also found in another key species, Persicobacter. (referred to here as denovo796; also from the Bacteroidetes) [79], which was correlated in the current study with the Gracilariafed sea urchins (Gracilaria-unique). However, it should be noted that low CAZyme content in a genome is not necessarily singular evidence for the non-engagement of a microbe in polysaccharide metabolism. A potential example gleaned from this study may be the core-generalist Fusibacter tunisiensis (referred to here as denovo 3738) which consists only of 7 CAZymes (with a total of 11 copies) but presents a relatively unique characteristic in terms of energy gain from the reduction of thiosulfate and elemental sulfur into sulfide. This metabolism is part of the sulfur cycle in anaerobic salty environments such as marine sediment [80], while in the currently examined sea urchins it may be attributed to the metabolism of the algal polysaccharides. This was evident in previous studies on decomposition of Ulva and Gracilaria where sulfate-reducing bacteria were noted for their major role in the process $[81,82]$.

While the above results revealed some supportive evidence for the specialization of the diet-unique microbes in terms of their unique metabolic content as well, it is still questionable whether encompassing a broad or narrow niche in the gut of T. gratilla elatensis is indicative of microbes' own metabolic arsenal (and vice versa). An interesting finding concerning this is the core microbe Candidatus hepatoplasma (a single OTU annotated as C. Hepatoplasma crinochetorum Av) identified here. This microbe, in spite of being fairly abundant in all examined niches of different diets and gut regions, reveals a relatively small genome of only 657,101 bp [83], no CAZymes, and also lacks a cell wall. This reinforces our previous assumption of this bacterium's host-dependent lifestyle in the gut of sea urchins. Another common microbe in $T$. gratilla elatensis gut was the generalist Gloeobacter kilaueensis (Cyanobacteria) which was found in all the niches examined. Such generalism in terms of a wide niche breadth can be correlated in the current study with the photoautotrophic lifestyle of this microbe [84] essential to its success in the sea urchin' light-penetrated gut, independent of the variation in available nutrients in the ingesta from different regions or diet types. The latter may be supported also by the relatively low content of CAZymes in this microbe's genome. In contrast to these two microbes, nearly all the other microbes that occupied broad niches, i.e., core, generalists, and coregeneralists, revealed a relatively rich carbohydrate metabolic capacity of between 14 and 81 CAZymes. Specialist microbes, with only two exceptions ( $R$. agariperforans and $U$. croceus), presented a relatively poorer carbohydrate metabolism potential consisting of between 7 and 13 enzyme classes (including sub-families). This finding may emphasize the correlation between a narrow niche breadth in terms of occupancy and occurrence, as measured for these microbes in the current study, and specification in the niche in terms of carrying only relevant enzymes for carbohydrates metabolism which are vital for gaining energy from the here examined diets.

\section{Conclusions}

The mono-specific algal diet altered the gut microbial assembly of $T$. gratilla elatensis in terms of composition, networking, and potential metabolism. A dense and well-connected microbial network was measured in the Ulva-fed sea urchins and may suggest, together with the animal's rapid growth, that this alga was most nutritious among the experimental diets. Generalism and 
specialism of the microbes in the gut agreed with their richness in CAZymes, while specialization in narrower niches of diet types was evident in terms of both occurrence and the specific metabolic arsenal the microbe carries that may aid the host in nutrition. Finally, we conclude Bacteroidetes to be the keystone phylum in the gut of $T$. gratilla elatensis with many different strains inhabiting this organ. Among are key hub microbes in the nichespecific networks, core microbes that occupied nearly all niches, generalist microbes that were suited to broader niches, as they are rich in their CAZyme content for decomposition of various polysaccharides, and specialist microbes that were correlated with the initial depolymerization stage in the metabolism of Ulva or Gracilaria.

\section{Abbreviations}

CAZy/CAZymes: Carbohydrate active enzymes; FDR: False discovery rate; GH: Glycoside hydrolase; GMA: Gut-microbe assembly; JEl: Jaccard edge index; NCM: National Center for Mariculture; PL: Polysaccharide lyase; QIIME: Quantitative insights into microbial ecology; NESH: Neighbor shift score; LEfSe: Linear discriminant analysis effect size; CCLasso: Correlation interference for compositional data through lasso; OTU: Operational taxonomic unit; PERMANOVA: Permutational multivariate analysis of variance.

\section{Supplementary Information}

The online version contains supplementary material available at https://doi. org/10.1186/s42523-021-00140-1.

\section{Additional file 1. Figure S1. The dissection process of sea urchin $T$.} gratilla elatensis for sampling of the three major regions of the digestive tract. Individual sea urchin (a); circumference cutting presenting upper and lower coelom (b); coelom after removal of the digestive tract (c); (d) Unfolded digestive tract presenting (left to right) the esophagus, stomach, and intestine regions. Red arrow indicates the Aristotle's lantern. Figure S2. Rarefaction curves present the observed OTUs in T. gratilla elatensis GMA from each dietary treatment and gut region $(n=51)$. Figure S3. Non-metric multidimensional scaling (NMDS) based on Bray-Curtis dissimilarities displaying GMA in different gut regions of T. gratilla elatensis fed with Gracilaria (a), algal-free pellets (b), or Ulva (c). Each dot represents GMA in a specific gut region in an individual sea urchin and is colored in red, green, and blue for esophagus, intestine, and stomach, respectively. The overall area of GMA in each gut region was measured following differences between individuals (inter-niche differences) and indicates overlaps between gut regions $(n=51)$. Figure S4. (a). A bubble chart of the 17 taxonomic phyla that were identified in the sea urchin digestive tract. The cumulative abundance of all OTUs of each phylum are represented as circles, each representing the sized cumulative abundance of the phylum OTUs in one individual sea urchin under a given treatment of diet and gut region. (b). Abundance of selected phyla with significant variations in the different examined niches of gut region and diet. Differences between individual samples in each specific niche are shown as box plots where middle line in the box indicates mean value, error lines present SD, and whiskers are drawn from the 10th to 90th percentiles $(n=54)$. Figure S5. Abundance/occurrence ratio of microbes identified as core, core generalists, generalists, specialists, or unique. Ratios of all individuals in group are summarized and presented as box plots with middle line for median, plus sign for mean, error lines for SD, and whiskers drawn from the 10th to 90 th percentiles $(n=50)$. Figure S6. Degrees distribution in GMA networks reveals the different distribution patterns (Poisson or Law-tail) of degrees between nodes in the microbe associations in the following niches: general network (a), under different diets of Gracilaria (b), pellets (c) or Ulva (d); or in different gut regions of esophagus (e), stomach (f), or intestine (g). Figure S7. Topology indices of the GMA association networks examined under different variables of diet (left) or gut region (right). Indices of the general network are shown in graph sets of different diets (left graphs). Figure S8. Schematic diagram of the associations of hub nodes in networks of different diets and gut regions. Type of association (co-occurrence or co-exclusion in blue or red lines, respectively) is also shown in cases of hub-hub association. Nodes are colored as per annotation at phyla level. Figure S9. Pairwise analysis of the association networks in different niches. Each network represents a network pair which was analyzed as control versus case in the respective order for network pairs: Gracilaria vs. pellet (a); Gracilaria vs. Ulva (b); Ulva vs. pellet (c); esophagus vs. stomach (d); esophagus vs. intestine (e); and stomach vs. esophagus (f); Edges in the combined network are colored as per their affiliation as exclusive to the control network (green), exclusive to case network (red), or shared between both (blue). Nodes in networks present only the identified driver microbes as measured by NetShift following their significant increased betweenness in the 'case' (red nodes) or control network (black nodes). Nodes diameter indicates NESH score from smaller to higher.

Table S1. Ingredients in the formulated algal-free, plant-based pellets for T. gratilla elatensis used in current study. Table S2. Physical characteristics of wet weight and diameter (mean \pm SE) of sea urchin individuals after culture under the different diet- types of UIva, or Gracilaria, or pellets. Values are mean $\pm S E(n=3)$. n.d. = not determined. Table S3. OTU count and number of reads for each of the examined samples from a particular gut region and diet regime $(n=54)$. Table S4. OTU count for the various identified phyla in sea urchin gut samples $(n=54)$. Table $\mathbf{5}$. Shared edges between GMA association networks in different diets or gut regions ( $n=54$ ) indicating type of relationship (co-occurrence or co-exclusion) and the participating nodes. Table S6. Topology properties of hub microbes in GMA association networks presenting number of degrees, closeness, and betweenness indices in the examined network of the different diets or gut regions $(n=54)$. Table S7. Topology properties of identified driver nodes in GMA association networks presenting Jaccard Edge Index, NESH score and delta betweenness centrality (normalized to the average path length) indices as measured in pairwise analyses of control versus case networks. Real drivers, i.e. nodes that were identified as driver in a specific niche against each of two other niches, are indicated in bold. Table S8. BLAST (Basic Local Alignment Search Tool) results of the closest phylogenetic species that was identified for each of the OTUs that were identified in current research as core, core-generalist, generalist, specialist, unique, or hub microbes. The bio project number in the NCBI database, where the genome of any of the assigned species is available, is provided. Not found $=$ no genome was available for the closest phylogeneticallyrelated species.

\section{Acknowledgements}

The authors would like to thank Dr. S. Green and team at RRC for the helpful advice prior to sequencing and analyses. We also thank the local staff at NCM, D. Ben-Ezra, V. Odintsov, L. Shauli, M. Fedyuk, and H. Chernova for all technical support and Mikhal Ben-Shaprut for English editing.

\section{Authors' contributions}

Conceptualization: LG; Investigation, Interpretation of results, and Visualization: MM, RB, AK, MS and LG; Funding acquisition: AG, NS, and LG; Project administration and Supervision: AK, and LG; Writing: MM, EKW, AK, and LG. All authors read and approved the final manuscript.

\section{Funding}

The current research was supported by The Jewish Charitable Association (JCA) and Israel Ministry of Energy Grant No. 215-11-032.

\section{Availability of data and materials}

The generated datasets in this study are accessible through ENA with the accession number PRJEB42313. Supplementary data and material are provided. 


\section{Declarations}

Ethics approval and consent to participate

Not applicable.

\section{Consent for publication}

Not applicable.

\section{Competing interests}

The authors declare that they have no competing interests.

\section{Author details}

${ }^{1}$ Marine Biology and Biotechnology Program, Department of Life Sciences, Ben-Gurion University of the Negev, Eilat Campus, Eilat, Israel. ${ }^{2}$ ssrael Oceanographic and Limnological Research, The National Center for Mariculture, P.O. Box 1212, 8811201 Eilat, Israel. ${ }^{3}$ Avram and Stella Goldstein-Goren, Department of Biotechnology Engineering, Ben-Gurion University of the Negev, P.O.B. 653, 8410501 Beer-Sheva, Israel. ${ }^{4}$ Morris Kahn Marine Research Station, The Leon H. Charney School of Marine Sciences, University of Haifa, 3498838 Haifa, Israel. ${ }^{5}$ Department of Environmental Studies, Tel Aviv University, P.O. Box 39040, 6997801 Tel Aviv, Israel. ${ }^{6}$ School of Mechanical Engineering, Tel Aviv University, P.O. Box 39040, 6997801 Tel Aviv, Israel.

Received: 21 April 2021 Accepted: 15 October 2021

Published online: 15 November 2021

\section{References}

1. Muller EE. Determining microbial niche breadth in the environment for better ecosystem fate predictions. MSystems. 2019;4(3):e00080-e119. https://doi.org/10.1128/mSystems.00080-19.

2. Sriswasdi S, Yang CC, Iwasaki W. Generalist species drive microbial dispersion and evolution. Nat Commun. 2017;8(1):1-8. https://doi.org/10.1038/ s41467-017-01265-1.

3. Liao J, Cao X, Zhao L, Wang J, Gao Z, Wang MC, Huang Y. The importance of neutral and niche processes for bacterial community assembly differs between habitat generalists and specialists. FEMS Microbiol Ecol. 2016. https://doi.org/10.1093/femsec/fiw174.

4. Layeghifard M, Hwang DM, Guttman DS. Disentangling interactions in the microbiome: a network perspective. Trends Microbiol. 2017;25(3):217-28. https://doi.org/10.1016/j.tim.2016.11.008.

5. Mitra K, Carvunis AR, Ramesh SK, Ideker T. Integrative approaches for finding modular structure in biological networks. Nat Rev Genet 2013;14(10):719-32. https://doi.org/10.1038/nrg3552.

6. Peura S, Bertilsson S, Jones RI, Eiler A. Resistant microbial cooccurrence patterns inferred by network topology. Appl Environ Microbiol. 2015:81(6):2090-7. https://doi.org/10.1128/AEM.03660-14.

7. Banerjee S, Schlaeppi K, van der Heijden MG. Keystone taxa as drivers of microbiome structure and functioning. Nat Rev Microbiol. 2018;16(9):567-76. https://doi.org/10.1038/s41579-018-0024-1.

8. Kuntal BK, Chandrakar P, Sadhu S, Mande SS. 'NetShift': a methodology for understanding 'driver microbes' from healthy and disease microbiome datasets. ISME J. 2019;13(2):442-54. https://doi.org/10.1038/ s41396-018-0291-X.

9. Harris JM. The presence, nature, and role of gut microflora in aquatic invertebrates: a synthesis. Microb Ecol. 1993;25(3):195-231. https://doi. org/10.1007/BF00171889.

10. Stimson J, Cunha T, Philippoff J. Food preferences and related behavior of the browsing sea urchin Tripneustes gratilla (Linnaeus) and its potential for use as a biological control agent. Mar Biol. 2007;151(5):1761-72. https:// doi.org/10.1007/s00227-007-0628-X.

11. Tuya F, Martín JA, Reuss GM, Luque A. Food preferences of the sea urchin Diadema antillarum in Gran Canaria (Canary Islands, central-east Atlantic Ocean). J Mar Biol Assoc UK. 2001;81(5):845-9. https://doi.org/10.1017/ S0025315401004672.

12. Shpigel M, Shauli L, Odintsov V, Ashkenazi N, Ben-Ezra D. Ulva lactuca biofilter from a land-based integrated multi trophic aquaculture (IMTA) system as a sole food source for the tropical sea urchin Tripneustes gratilla elatensis. Aquaculture. 2018;496:221-31. https://doi.org/10.1016/j.aquac ulture.2018.06.038.

13. Shpigel M, McBride SC, Marciano S, Ron S, Ben-Amotz A. Improving gonad colour and somatic index in the European sea urchin Paracentrotus lividus. Aquaculture. 2005;245(1-4):101-9. https://doi.org/10.1016/j. aquaculture.2004.11.043.

14. Shpigel M, Erez J. Effect of diets and light regimes on calcification and somatic growth of the sea urchin Tripneustes gratilla elatensis. Aquaculture. 2020;529: 735547. https://doi.org/10.1016/j.aquaculture.2020. 735547.

15. Duckworth M, Hong KC, Yaphe W. The agar polysaccharides of Gracilaria species. Carbohyd Res. 1971;18(1):1-9. https://doi.org/10.1016/S00086215(00)80253-0.

16. Lahaye $M$, Robic A. Structure and functional properties of ulvan, a polysaccharide from green seaweeds. Biomacromol. 2007;8(6):1765-74. https://doi.org/10.1021/bm061185q.

17. Holland ND. Digestive system. In: Lawrence JM Sea urchins: biology and ecology. UK; 2013. p. 119-133. https://doi.org/10.1016/B978-0-12396491-5.00008-3.

18. Holland ND, Ghiselin MT. A comparative study of gut mucous cells in thirty-seven species of the class Echinoidea (Echinodermata). Biol Bull. 1970;138(3):286-305.

19. Trenzado CE, Hidalgo F, Villanueva D, Furné M, Díaz-Casado ME, Merino $R$, Sanz A. Study of the enzymatic digestive profile in three species of Mediterranean sea urchins. Aquaculture. 2012;344:174-80. https://doi. org/10.1016/j.aquaculture.2012.03.027.

20. Bishop CD, Watts SA. Biochemical and morphometric study of growth in the stomach and intestine of the echinoid Lytechinus variegatus (Echinodermata). Mar Biol. 1992;114(3):459-67. https://doi.org/10.1007/BF003 50038 .

21. Walters W, Hyde ER, Berg-Lyons D, Ackermann G, Humphrey G, Parada A, Gilbert JA, Jansson JK, Caporaso JG, Fuhrman JA, Apprill A. Improved bacterial 16S rRNA gene (V4 and V4-5) and fungal internal transcribed spacer marker gene primers for microbial community surveys. Msystems. 2016;1(1):e00009-15. https://doi.org/10.1128/mSystems.00009-15.

22. Caporaso JG, Lauber CL, Walters WA, Berg-Lyons D, Huntley J, Fierer N, Owens SM, Betley J, Fraser L, Bauer M, Gormley N. Ultra-high-throughput microbial community analysis on the Illumina HiSeq and MiSeq platforms. ISME J. 2012;6(8):1621-4. https://doi.org/10.1038/ismej.2012.8.

23. Caporaso JG, Kuczynski J, Stombaugh J, Bittinger K, Bushman FD, Costello EK, Fierer N, Peña AG, Goodrich JK, Gordon Jl, Huttley GA. QIIME allows analysis of high-throughput community sequencing data. Nat Methods. 2010;7(5):335-6. https://doi.org/10.1038/nmeth.f.303.

24. Zhang J, Kobert K, Flouri T, Stamatakis A. PEAR: a fast and accurate illumina paired-end reAd mergeR. Bioinformatics. 2014;30(5):614-20. https:// doi.org/10.1093/bioinformatics/btt593.

25. Edgar RC. Search and clustering orders of magnitude faster than BLAST. Bioinformatics. 2010;26(19):2460-1. https://doi.org/10.1093/bioinforma tics/btq461.

26. Quast C, Pruesse E, Yilmaz P, Gerken J, Schweer T, Yarza P, Peplies J, Glöckner FO. The SILVA ribosomal RNA gene database project: improved data processing and web-based tools. Nucleic Acids Res. 2012;41(D1):D590-6. https://doi.org/10.1093/nar/gks1219.

27. Edgar RC, Haas BJ, Clemente JC, Quince C, Knight R. UCHIME improves sensitivity and speed of chimera detection. Bioinformatics. 2011;27(16):2194-200. https://doi.org/10.1093/bioinformatics/btr381.

28. Caporaso JG, Bittinger K, Bushman FD, DeSantis TZ, Andersen GL, Knight R. PyNAST: a flexible tool for aligning sequences to a template alignment. Bioinformatics. 2010;26(2):266-7. https://doi.org/10.1093/bioinformatics/ btp636.

29. Segata N, Izard J, Waldron L, Gevers D, Miropolsky L, Garrett WS, Huttenhower C. Metagenomic biomarker discovery and explanation. Genome Biol. 2011;12(6):1-8. https://doi.org/10.1186/gb-2011-12-6-r60.

30. Kokou F, Sasson G, Friedman J, Eyal S, Ovadia O, Harpaz S, Cnaani A, Mizrahi I. Core gut microbial communities are maintained by beneficial interactions and strain variability in fish. Nat Microbiol. 2019;4(12):245665. https://doi.org/10.1038/s41564-019-0560-0.

31. Shannon CE, Weaver W. The mathematical theory of communication. Urbana-Champaign: University of Illinois Press, p. 117. 
32. Fox BJ. Niche parameters and species richness. Ecology. 1981;62(6):141525. https://doi.org/10.2307/1941497.

33. Levins R. Evolution in changing environments. Princeton University Press; 2020. https://doi.org/10.1515/9780691209418.

34. Nagpal S, Singh R, Yadav D, Mande SS. MetagenoNets: comprehensive inference and meta-insights for microbial correlation networks. Nucleic Acids Res. 2020:48(1):572-9. https://doi.org/10.1093/nar/gkaa254.

35. Fang $\mathrm{H}$, Huang $\mathrm{C}$, Zhao H, Deng M. CCLasso: correlation inference for compositional data through Lasso. Bioinformatics. 2015;31(19):3172-80. https://doi.org/10.1093/bioinformatics/btv349.

36. Shannon P, Markiel A, Ozier O, Baliga NS, Wang JT, Ramage D, Amin N, Schwikowski B, Ideker T. Cytoscape: a software environment for integrated models of biomolecular interaction networks. Genome Res. 2003;13(11):2498-504. https://doi.org/10.1101/gr.1239303.

37. Barabási AL, Albert R. Emergence of scaling in random networks. Science. 1999;286(5439):509-12. https://doi.org/10.1126/science.286.5439.509.

38. Jalili M, Salehzadeh-Yazdi A, Asgari Y, Arab SS, Yaghmaie M, Ghavamzadeh A, Alimoghaddam K. CentiServer: a comprehensive resource, web-based application and R package for centrality analysis. PLOS ONE. 2015;10(11):e0143111. https://doi.org/10.1371/journal.pone.0143111.

39. Kleinberg JM. Hubs, authorities, and communities. ACM Comput Surv. 1999:31(4):5.

40. Berry D, Widder S. Deciphering microbial interactions and detecting keystone species with co-occurrence networks. Front Microbiol. 2014;5:219. https://doi.org/10.3389/fmicb.2014.00219.

41. Johnson M, Zaretskaya I, Raytselis Y, Merezhuk Y, McGinnis S, Madden TL. NCBI BLAST: a better web interface. Nucleic Acids Res. 2008;36:5-9. https://doi.org/10.1093/nar/gkn201.

42. Zhang H, Yohe T, Huang L, Entwistle S, Wu P, Yang Z, Busk PK, Xu Y, Yin Y. dbCAN2: a meta server for automated carbohydrate-active enzyme annotation. Nucleic Acids Res. 2018;46(1):95-101. https://doi.org/10. 1093/nar/gky418

43. Dhariwal A, Chong J, Habib S, King IL, Agellon LB, Xia J. MicrobiomeAnalyst: a web-based tool for comprehensive statistical, visual and metaanalysis of microbiome data. Nucleic Acids Res. 2017;45(1):180-8. https:// doi.org/10.1093/nar/gkx295.

44. Chong J, Liu P, Zhou G, Xia J. Using Microbiome analyst for comprehensive statistical, functional, and meta-analysis of microbiome data. Nat Protoc. 2020;15(3):799-821. https://doi.org/10.1038/s41596-019-0264-1.

45. Hakim JA, Koo H, Dennis LN, Kumar R, PtacekT, Morrow CD, Lefkowitz EJ, Powell ML, Bej AK, Watts SA. An abundance of Epsilonproteobacteria revealed in the gut microbiome of the laboratory cultured sea urchin Lytechinus variegatus. Front Microbiol. 2015;6:1047. https://doi.org/10. 3389/fmicb.2015.01047.

46. Hakim JA, Koo H, Kumar R, Lefkowitz EJ, Morrow CD, Powell ML, Watts SA, Bej AK. The gut microbiome of the sea urchin, Lytechinus variegatus, from its natural habitat demonstrates selective attributes of microbial taxa and predictive metabolic profiles. FEMS Microbiol Ecol. 2016. https://doi.org/ 10.1093/femsec/fiw146.

47. Lawrence JM, Lawrence AL, Watts SA. Feeding, digestion and digestibility of sea urchins. Dev Aquacult Fish Sci. 2013;38:135-54. https://doi.org/10. 1016/B978-0-12-396491-5.00009-5.

48. Thorsen MS. Microbial activity, oxygen status and fermentation in the gut of the irregular sea urchin Echinocardium cordatum (Spatangoida: Echinodermata). Mar Biol. 1998;132(3):423-33. https://doi.org/10.1007/ s002270050408.

49. Ziegler A, Faber C, Mueller S, Bartolomaeus T. Systematic comparison and reconstruction of sea urchin (Echinoidea) internal anatomy: a novel approach using magnetic resonance imaging. BMC Biol. 2008;6(1):1-5. https://doi.org/10.1186/1741-7007-6-33.

50. Risely A. Applying the core microbiome to understand host-microbe systems. J Anim Ecol. 2020;89(7):1549-58. https://doi.org/10.1111/13652656.13229.

51. Shade A, Handelsman J. Beyond the Venn diagram: the hunt for a core microbiome. Environ Microbiol. 2012;14(1):4-12. https://doi.org/10. 1111/j.1462-2920.2011.02585.x.

52. Hakim JA, Schram JB, Galloway AW, Morrow CD, Crowley MR, Watts SA, Bej AK. The purple sea urchin Strongylocentrotus purpuratus demonstrates a compartmentalization of gut bacterial microbiota, predictive functional attributes, and taxonomic co-occurrence. Microorganisms. 2019;7(2):35. https://doi.org/10.3390/microorganisms7020035.
53. Yao Q, Yu K, Liang J, Wang Y, Hu B, Huang X, Chen B, Qin Z. The composition, diversity and predictive metabolic profiles of bacteria associated with the gut digesta of five sea urchins in Luhuitou fringing reef (northern South China Sea). Front Microbiol. 2019;10:1168. https://doi.org/10. 3389/fmicb.2019.01168.

54. Tanrattanapitak N, Pairohakul S. Bacterial community in gut contents of the sea urchin Diadema setosum (Leske, 1778) and the ambient sediments from Sichang Island using metagenomics approaches. Int I Sci. 2018;15(1):117-25.

55. Thomas F, Hehemann JH, Rebuffet E, Czjzek M, Michel G. Environmental and gut bacteroidetes: the food connection. Front Microbiol. 2011;2:93. https://doi.org/10.3389/fmicb.2011.00093.

56. Hollants J, Leliaert F, De Clerck O, Willems A. What we can learn from sushi: a review on seaweed-bacterial associations. FEMS Microbiol Ecol. 2013;83(1):1-6. https://doi.org/10.1111/j.1574-6941.2012.01446.x.

57. Egan S, Harder T, Burke C, Steinberg P, Kjelleberg S, Thomas T. The seaweed holobiont: understanding seaweed-bacteria interactions. FEMS Microbiol Rev. 2013;37(3):462-76. https://doi.org/10.1111/1574-6976. 12011.

58. Doezemcl P. Carbohydrates and carbohydrate metabolism of echinoderms. Chem Zool V3 Echinnodermata Nematoda Acanthocephala. 2012;101.

59. Strogatz SH. Exploring complex networks. Nature. 2001;410(6825):26876. https://doi.org/10.1038/35065725.

60. Ma B, Wang Y, Ye S, Liu S, Stirling E, Gilbert JA, Faust K, Knight R, Jansson $J K$, Cardona C, Röttjers L. Earth microbial co-occurrence network reveals interconnection pattern across microbiomes. Microbiome. 2020;8:1-2. https://doi.org/10.1186/s40168-020-00857-2.

61. Barmpoutis D, Murray RM. Networks with the smallest average distance and the largest average clustering. arXiv preprint arXiv: 1007.4031. 2010.

62. Zelezniak A, Andrejev S, Ponomarova O, Mende DR, Bork P, Patil KR. Metabolic dependencies drive species co-occurrence in diverse microbial communities. Proc Natl Acad Sci. 2015;112(20):6449-54. https://doi.org/ 10.1073/pnas.1421834112.

63. Louca S, Polz MF, Mazel F, Albright MB, Huber JA, O'Connor MI, Ackermann M, Hahn AS, Srivastava DS, Crowe SA, Doebeli M. Function and functional redundancy in microbial systems. Nat Ecol Evolut. 2018;2(6):936-43. https://doi.org/10.1038/s41559-018-0519-1.

64. Shade A, Peter H, Allison SD, Baho D, Berga M, Bürgmann H, Huber DH, Langenheder S, Lennon JT, Martiny JB, Matulich KL. Fundamentals of microbial community resistance and resilience. Front Microbiol. 2012;3:417. https://doi.org/10.3389/fmicb.2012.00417.

65. Yu-Qing T, Mahmood K, Shehzadi R, Ashraf MF. Ulva lactuca and its polysaccharides: Food and biomedical aspects. J Biol Agric Healthcare. 2016;6(1):140-51

66. Melo MR, Feitosa JP, Freitas AL, De Paula RC. Isolation and characterization of soluble sulfated polysaccharide from the red seaweed Gracilaria cornea. Carbohydr Polym. 2002;49(4):491-8. https://doi.org/10.1016/ S0144-8617(02)00006-1.

67. Moraiis S, Mizrahi I. Islands in the stream: from individual to communal fiber degradation in the rumen ecosystem. FEMS Microbiol Rev. 2019;43(4):362-79. https://doi.org/10.1093/femsre/fuz007.

68. Konopka A, Lindemann S, Fredrickson J. Dynamics in microbial communities: unraveling mechanisms to identify principles. ISME J. 2015;9(7):148895. https://doi.org/10.1038/ismej.2014.251.

69. Kohl KD, Skopec MM, Dearing MD. Captivity results in disparate loss of gut microbial diversity in closely related hosts. Conserv Physiol. 2014;2(1):cou009. https://doi.org/10.1093/conphys/cou009.

70. Dhanasiri AK, Brunvold L, Brinchmann MF, Korsnes K, Bergh O, Kiron V. Changes in the intestinal microbiota of wild Atlantic cod Gadus morhua L. upon captive rearing. Microbial Ecol. 2011;61(1):20-30. https://doi.org/10. 1007/s00248-010-9673-y.

71. Bell TH, Bell T. Many roads to bacterial generalism. FEMS Microbiol Ecol. 2021:97(1):240. https://doi.org/10.1093/femsec/fiaa240.

72. Parente E, Zotta T, Faust K, De Filippis F, Ercolini D. Structure of association networks in food bacterial communities. Food Microbiol. 2018;73:49-60. https://doi.org/10.1016/j.fm.2017.12.010

73. Konur O. Glycoscience: the current state of the research. In: Marine glycobiology. CRC Press;2016. p. 27-42.

74. Qu L, Zhu F, Hong X, Gao W, Chen J, Sun X. Sunxiuqinia elliptica gen. nov., $s p$, nov., a member of the phylum Bacteroidetes isolated from sediment in 
a sea cucumber farm. Int J System Evolut Microbiol. 2011;61 (12):2885-9. https://doi.org/10.1099/ijs.0.026971-0.

75. Meziti A, Kormas KA, Pancucci-Papadopoulou MA, Thessalou-Legaki M. Bacterial phylotypes associated with the digestive tract of the sea urchin Paracentrotus lividus and the ascidian Microcosmus sp. Russ J Mar Biol. 2007;33(2):84-91. https://doi.org/10.1134/S1063074007020022.

76. Schwob G, Cabrol L, Poulin E, Orlando J. Characterization of the gut microbiota of the Antarctic heart urchin (Spatangoida) Abatus agassizii. Front Microbiol. 2020:11:308. https://doi.org/10.3389/fmicb.2020.00308.

77. Reisky L, Prechoux A, Zühlke MK, Bäumgen M, Robb CS, Gerlach N, Roret T, Stanetty C, Larocque R, Michel G, Song T. A marine bacterial enzymatic cascade degrades the algal polysaccharide ulvan. Nat Chem Biol. 2019;15(8):803-12. https://doi.org/10.1038/s41589-019-0311-9.

78. Fu XT, Kim SM. Agarase: review of major sources, categories, purification method, enzyme characteristics and applications. Mar Drugs. 2010;8(1):200-18. https://doi.org/10.3390/md8010200

79. Furusawa G, Lau NS, Suganthi A, Amirul AA. Agarolytic bacterium Persicobacter sp. CCB-QB 2 exhibited a diauxic growth involving galactose utilization pathway. MicrobiologyOpen. 2017;6(1):e00405. https://doi.org/ $10.1002 / \mathrm{mbo3} .405$

80. Jørgensen BB, Findlay AJ, Pellerin A. The biogeochemical sulfur cycle of marine sediments. Front Microbiol. 2019;10:849. https://doi.org/10.3389/ fmicb.2019.00849.
81. Marinho-Soriano E. Agar polysaccharides from Gracilaria species (Rhodophyta, Gracilariaceae). J Biotechnol. 2001;89(1):81-4. https://doi.org/10. 1016/S0168-1656(01)00255-3.

82. Nedergaard Rl, Risgaard-Petersen N, Finster K. The importance of sulfate reduction associated with Ulva lactuca thalli during decomposition: a mesocosm experiment. J Exp Mar Biol Ecol. 2002;275(1):15-29. https:// doi.org/10.1016/S0022-0981(02)00211-3.

83. Wang Y, Stingl U, Anton-Erxleben F, Geisler S, Brune A, Zimmer M. "Candidatus Hepatoplasma crinochetorum", a new, stalk-forming lineage of Mollicutes colonizing the midgut glands of a terrestrial isopod. Appl Environ Microbiol. 2004;70(10):6166-72. https://doi.org/10.1128/AEM.70. 10.6166-6172.2004.

84. Saw JH, Schatz M, Brown MV, Kunkel DD, Foster JS, Shick H, Christensen S, Hou S, Wan X, Donachie SP. Cultivation and complete genome sequencing of Gloeobacter kilaueensis sp. nov., from a lava cave in Kilauea Caldera, Hawai'i. PLoS ONE. 2013;8(10):76376. https://doi.org/10.1371/journal. pone.0076376.

\section{Publisher's Note}

Springer Nature remains neutral with regard to jurisdictional claims in published maps and institutional affiliations.
Ready to submit your research? Choose BMC and benefit from:

- fast, convenient online submission

- thorough peer review by experienced researchers in your field

- rapid publication on acceptance

- support for research data, including large and complex data types

- gold Open Access which fosters wider collaboration and increased citations

- maximum visibility for your research: over 100M website views per year

At BMC, research is always in progress.

Learn more biomedcentral.com/submissions 